\title{
Synthesis of the C4-C16 Polyketide Fragment of Portimines A and B
}

Xiao-Bo Ding, ${ }^{\dagger}$ Harry R. M. Aitken, ${ }^{\dagger}$ Esperanza S. Pearl, ${ }^{\dagger}$ Daniel P. Furkert, ${ }^{*,+,}$ Margaret A. Brimble ${ }^{*,+, \ddagger}$

† School of Chemical Sciences, The University of Auckland, 23 Symonds Street, Auckland, 1010, New Zealand ¥The Maurice Wilkins Centre for Molecular Biodiscovery, The University of Auckland, 3 Symonds Street, Auckland, 1010, New Zealand

Copies of ${ }^{1} \mathrm{H},{ }^{13} \mathrm{C}\left\{{ }^{1} \mathrm{H}\right\}$ NMR spectra of compound 20

Copies of ${ }^{1} \mathrm{H},{ }^{13} \mathrm{C}\left\{{ }^{1} \mathrm{H}\right\}$ NMR spectra of compound S1 S3

Copies of ${ }^{1} \mathrm{H},{ }^{13} \mathrm{C}\left\{{ }^{1} \mathrm{H}\right\}$ NMR spectra of compound $\mathbf{1 7}$

Copies of ${ }^{1} \mathrm{H},{ }^{13} \mathrm{C}\left\{{ }^{1} \mathrm{H}\right\}$ NMR spectra of compound 22

Copies of ${ }^{1} \mathrm{H},{ }^{13} \mathrm{C}\left\{{ }^{1} \mathrm{H}\right\}$ NMR spectra of compound $24 \quad$ S6

Copies of ${ }^{1} \mathrm{H},{ }^{13} \mathrm{C}\left\{{ }^{1} \mathrm{H}\right\}$ NMR spectra of compound S2

Copies of ${ }^{1} \mathrm{H},{ }^{13} \mathrm{C}\left\{{ }^{1} \mathrm{H}\right\}$ NMR spectra of compound S3 S8

Copies of ${ }^{1} \mathrm{H},{ }^{13} \mathrm{C}\left\{{ }^{1} \mathrm{H}\right\}$ NMR spectra of compound 25

Copies of ${ }^{1} \mathrm{H},{ }^{13} \mathrm{C}\left\{{ }^{1} \mathrm{H}\right\}$ NMR spectra of compound $26 \quad \mathrm{~S} 10$

Copies of ${ }^{1} \mathrm{H},{ }^{13} \mathrm{C}\left\{{ }^{1} \mathrm{H}\right\}$ NMR spectra of compound $\mathbf{S 4} \quad \mathrm{S} 11$

Copies of ${ }^{1} \mathrm{H},{ }^{13} \mathrm{C}\left\{{ }^{1} \mathrm{H}\right\}$ NMR spectra of compound $28 \quad \mathrm{~S} 12$

Copies of ${ }^{1} \mathrm{H},{ }^{13} \mathrm{C}\left\{{ }^{1} \mathrm{H}\right\}$ NMR spectra of compound $29 \quad \mathrm{~S} 13$

Copies of ${ }^{1} \mathrm{H},{ }^{13} \mathrm{C}\left\{{ }^{1} \mathrm{H}\right\}$ NMR spectra of compound $31 \quad \mathrm{~S} 14$

Copies of ${ }^{1} \mathrm{H},{ }^{13} \mathrm{C}\left\{{ }^{1} \mathrm{H}\right\}$ NMR spectra of compound $36 \quad \mathrm{~S} 15$

Copies of ${ }^{1} \mathrm{H},{ }^{13} \mathrm{C}\left\{{ }^{1} \mathrm{H}\right\}$ NMR spectra of compound $\mathbf{S 5} \quad \mathrm{S} 16$

Copies of ${ }^{1} \mathrm{H},{ }^{13} \mathrm{C}\left\{{ }^{1} \mathrm{H}\right\}$ NMR spectra of compound $37 \quad \mathrm{~S} 17$

Copies of ${ }^{1} \mathrm{H},{ }^{13} \mathrm{C}\left\{{ }^{1} \mathrm{H}\right\}$ NMR spectra of compound $38 \quad \mathrm{~S} 18$

Copies of ${ }^{1} \mathrm{H},{ }^{13} \mathrm{C}\left\{{ }^{1} \mathrm{H}\right\}$ NMR spectra of compound $\mathbf{1 4} \quad \mathrm{S} 19$

Copies of ${ }^{1} \mathrm{H},{ }^{13} \mathrm{C}\left\{{ }^{1} \mathrm{H}\right\}$ NMR spectra of compound $\mathbf{1 3} \quad \mathrm{S} 20$

Copies of ${ }^{1} \mathrm{H},{ }^{13} \mathrm{C}\left\{{ }^{1} \mathrm{H}\right\}$ NMR spectra of compound $39 \quad$ S21

Copies of ${ }^{1} \mathrm{H},{ }^{13} \mathrm{C}\left\{{ }^{1} \mathrm{H}\right\}$ NMR spectra of compound $\mathbf{1 2} \quad$ S22

Copies of ${ }^{1} \mathrm{H},{ }^{13} \mathrm{C}\left\{{ }^{1} \mathrm{H}\right\}$ NMR spectra of compound $\mathbf{4 0} \quad \mathrm{S} 23$

Copies of ${ }^{1} \mathrm{H},{ }^{13} \mathrm{C}\left\{{ }^{1} \mathrm{H}\right\}$ NMR spectra of compound S6 $\quad$ S24

Copies of ${ }^{1} \mathrm{H},{ }^{13} \mathrm{C}\left\{{ }^{1} \mathrm{H}\right\}$ NMR spectra of compound $\mathbf{4 2} \quad \mathrm{S} 25$

Determination of the enantiomeric excess of crotylation product $\mathbf{2 4}$ S26

Conditions attempted for the cyclisation of compound $\mathbf{4 0 .} \quad$ S30 

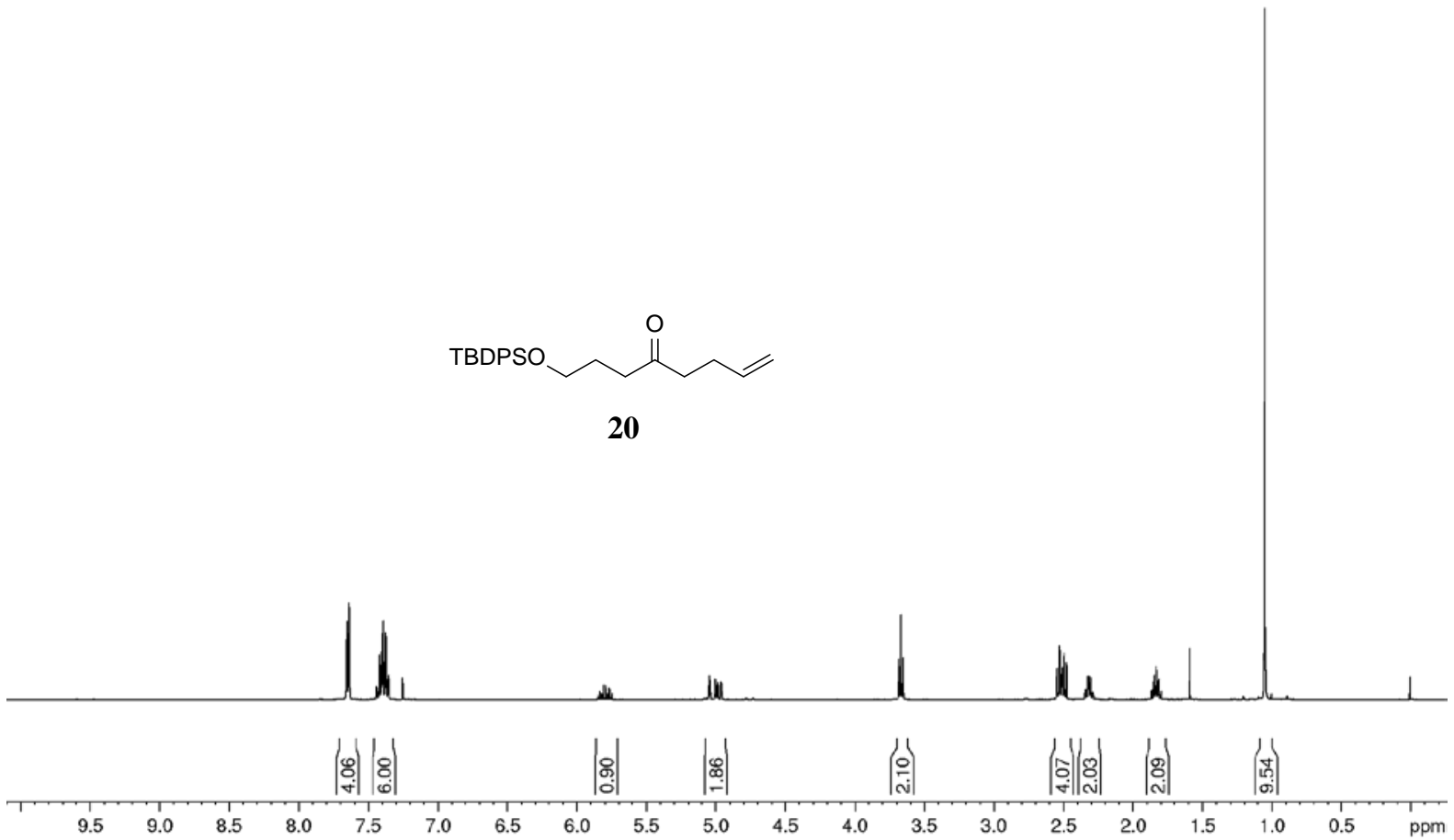

Compound 20, ${ }^{13} \mathrm{C}\left\{{ }^{1} \mathrm{H}\right\} \mathrm{NMR}\left(\mathrm{CDCl}_{3}, 100 \mathrm{MHz}\right)$

||

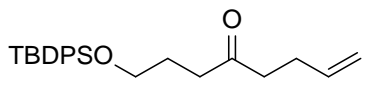

20
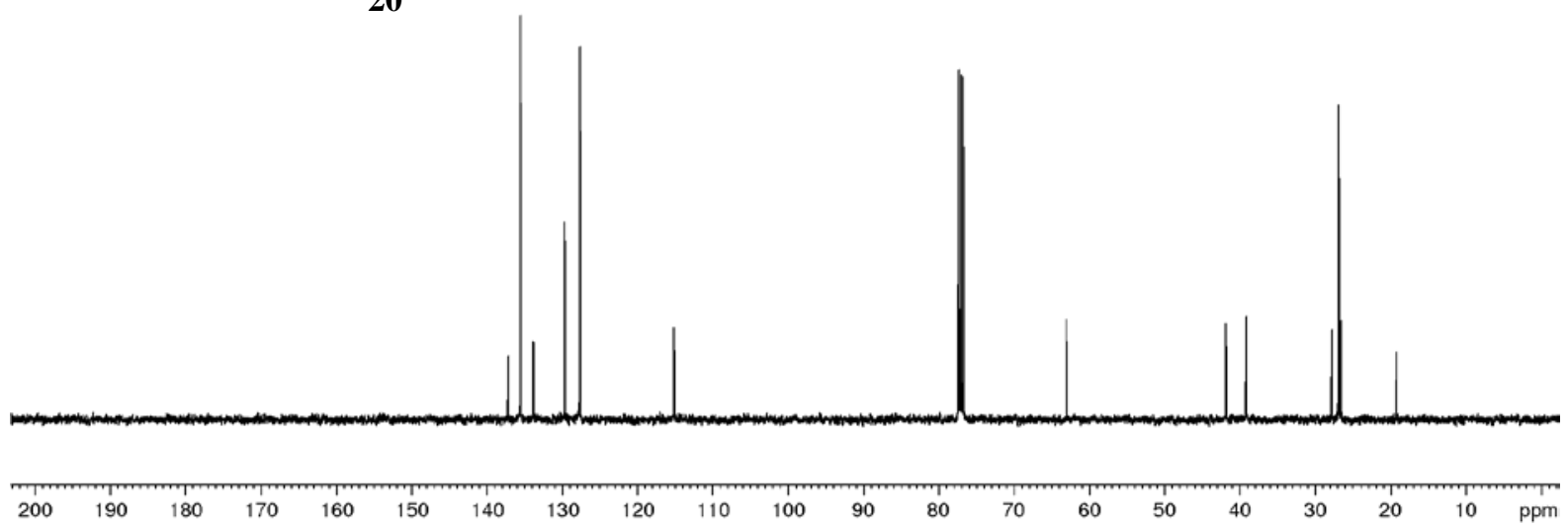

90
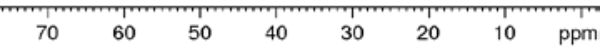
Compound S1, ${ }^{1} \mathrm{H}$ NMR ( $\left.\mathrm{CDCl}_{3}, 400 \mathrm{MHz}\right)$
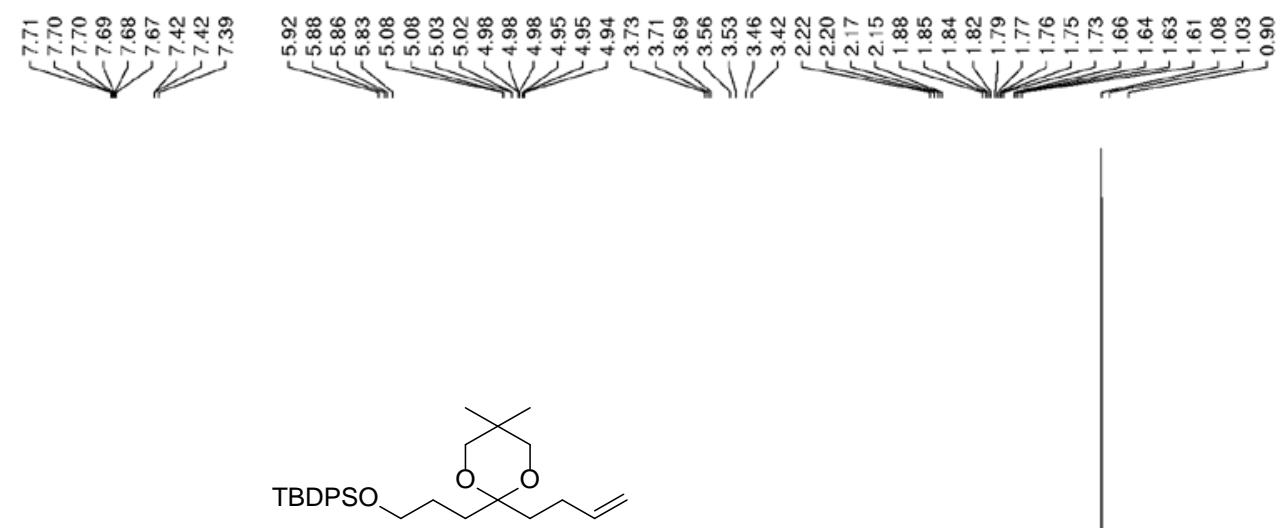

S1
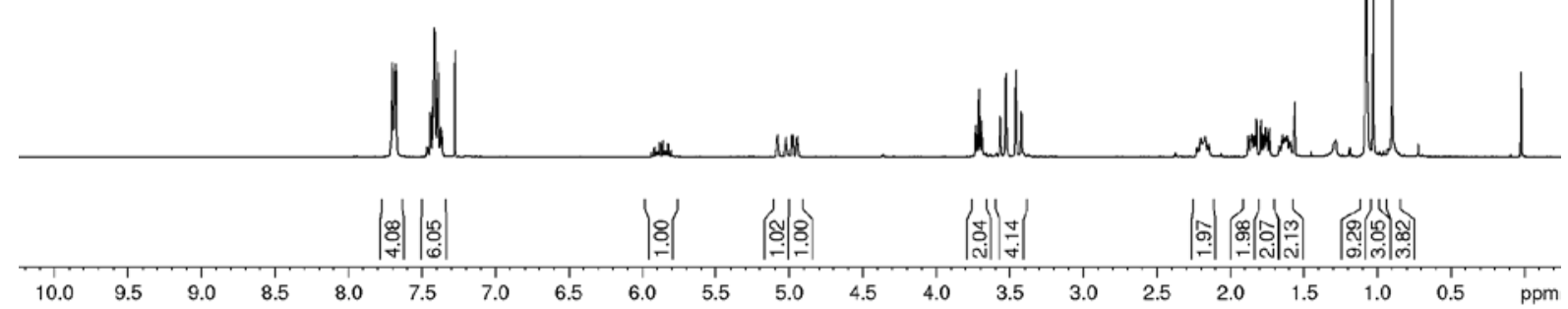

Compound S1, ${ }^{13} \mathrm{C}\left\{{ }^{1} \mathrm{H}\right\}$ NMR $\left(\mathrm{CDCl}_{3}, 100 \mathrm{MHz}\right)$

l1/
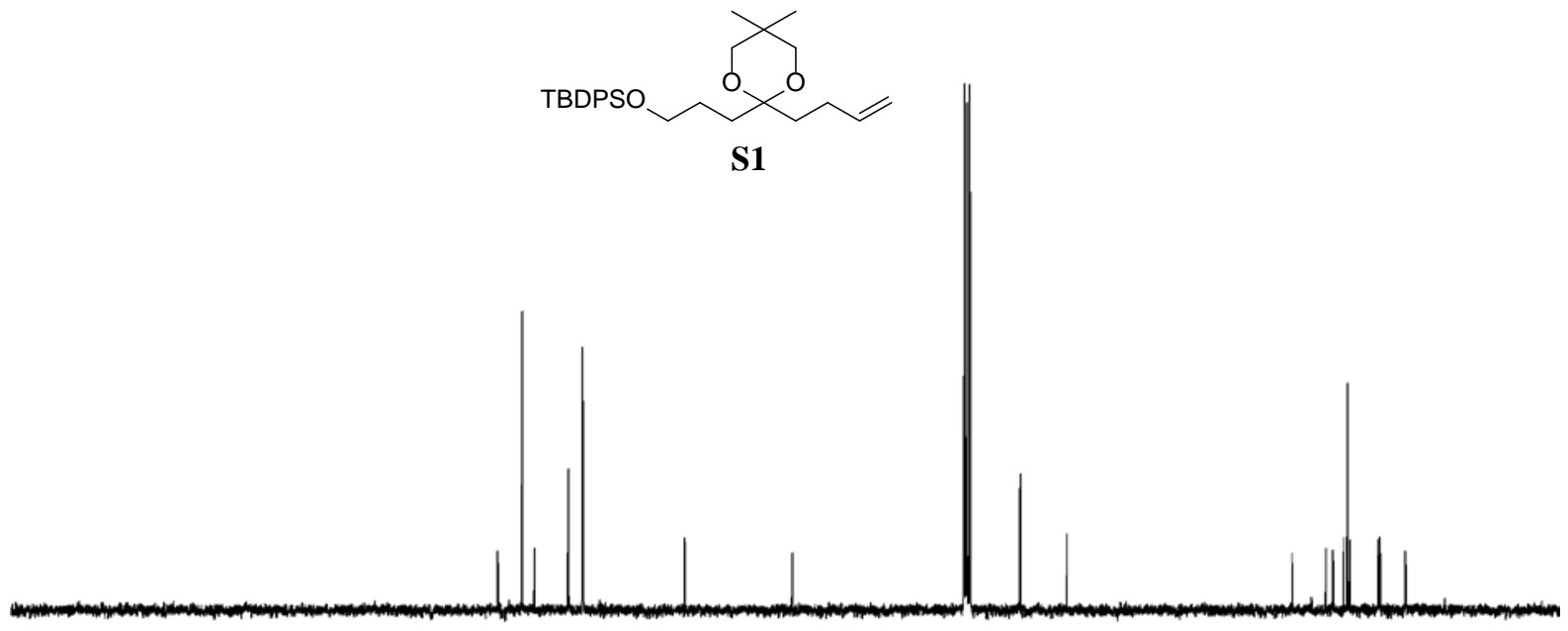

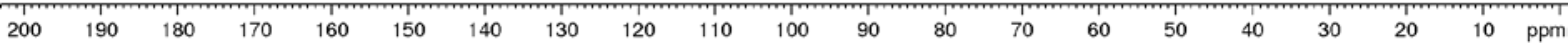


Compound 17, ${ }^{1} \mathrm{H}$ NMR $\left(\mathrm{CDCl}_{3}, 400 \mathrm{MHz}\right)$
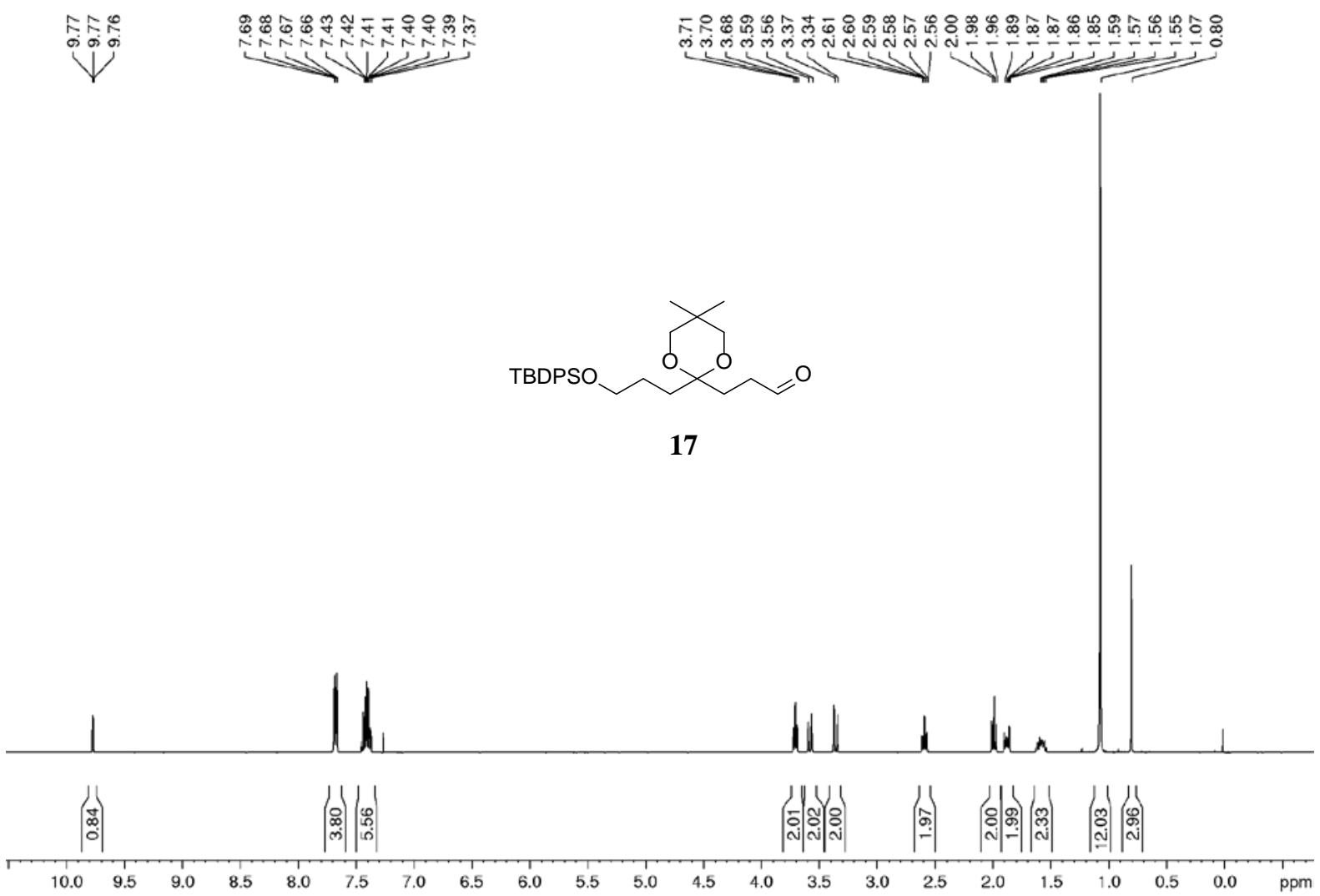

Compound 17, ${ }^{13} \mathrm{C}\left\{{ }^{1} \mathrm{H}\right\} \mathrm{NMR}\left(\mathrm{CDCl}_{3}, 100 \mathrm{MHz}\right)$
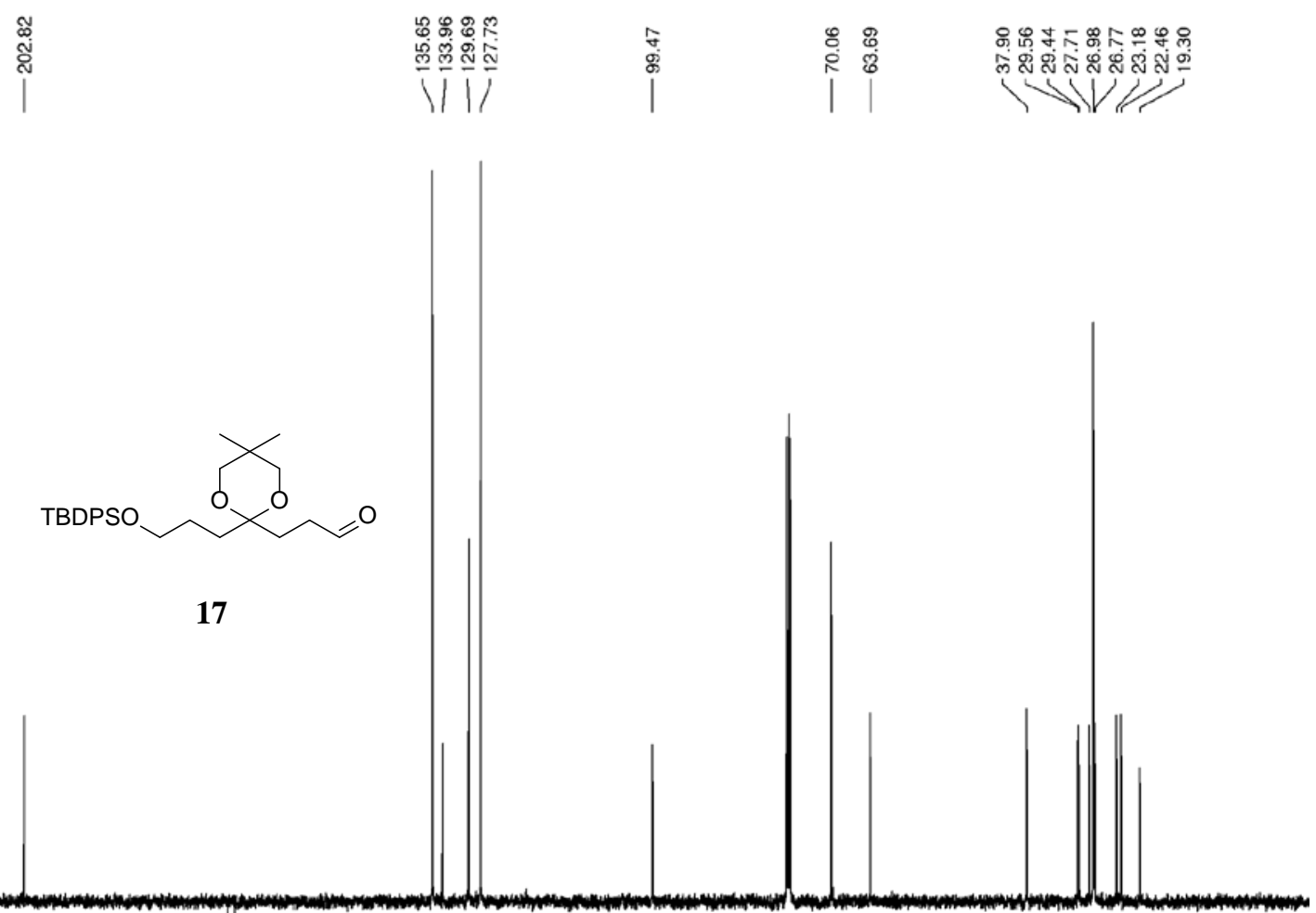

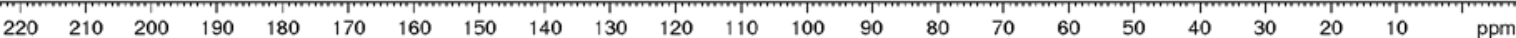




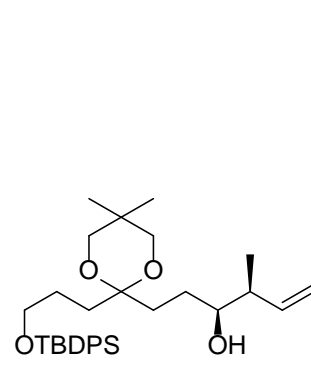

22
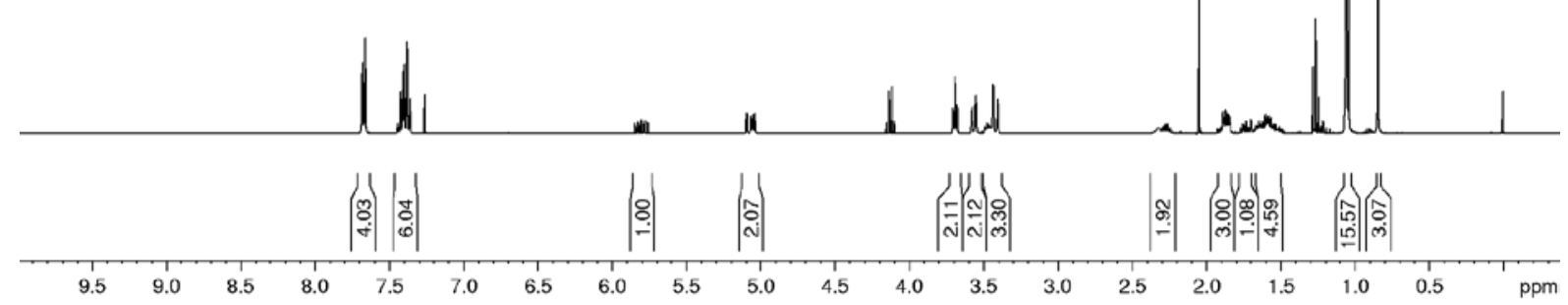

Compound 22, ${ }^{13} \mathrm{C}\left\{{ }^{1} \mathrm{H}\right\}$ NMR $\left(\mathrm{CDCl}_{3}, 100 \mathrm{MHz}\right)$

|
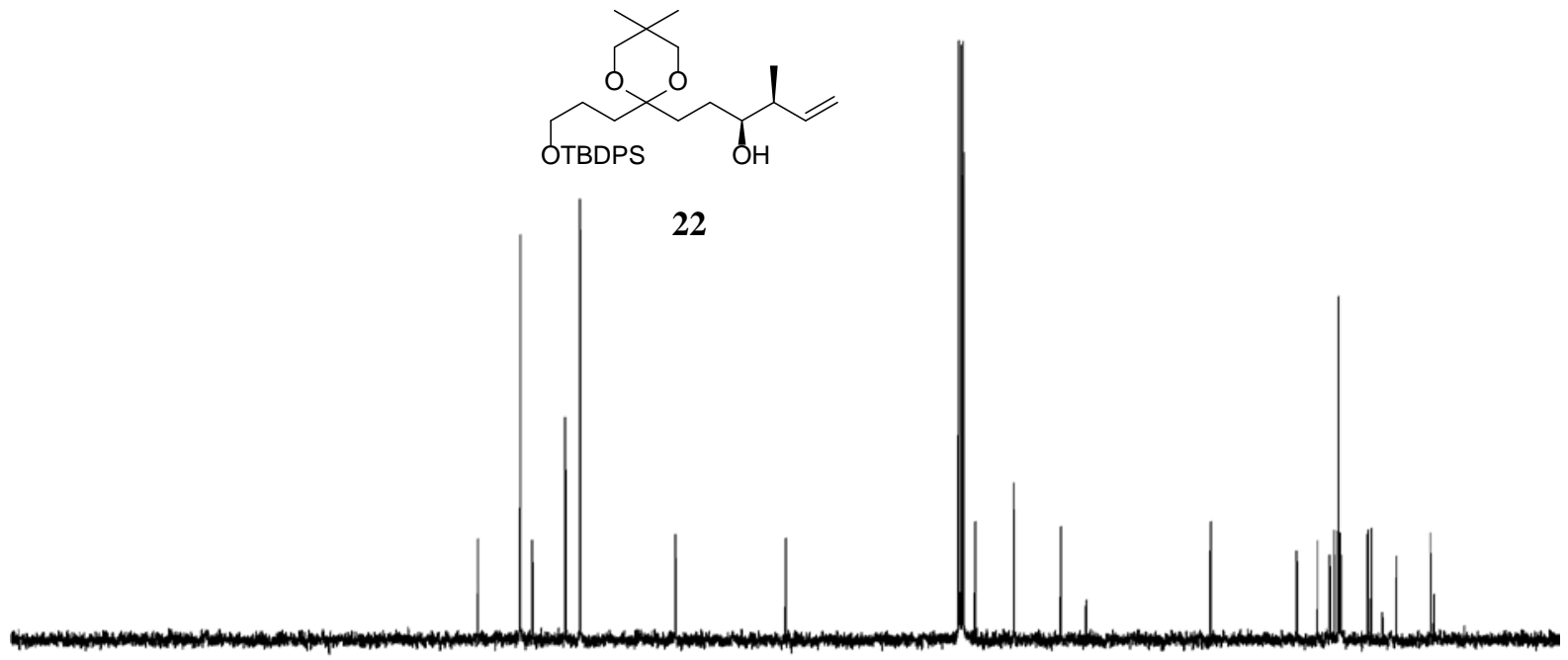

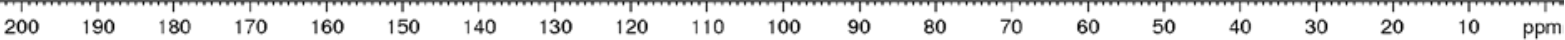


Compound 24, ${ }^{1} \mathrm{H} \mathrm{NMR}\left(\mathrm{CDCl}_{3}, 400 \mathrm{MHz}\right)$

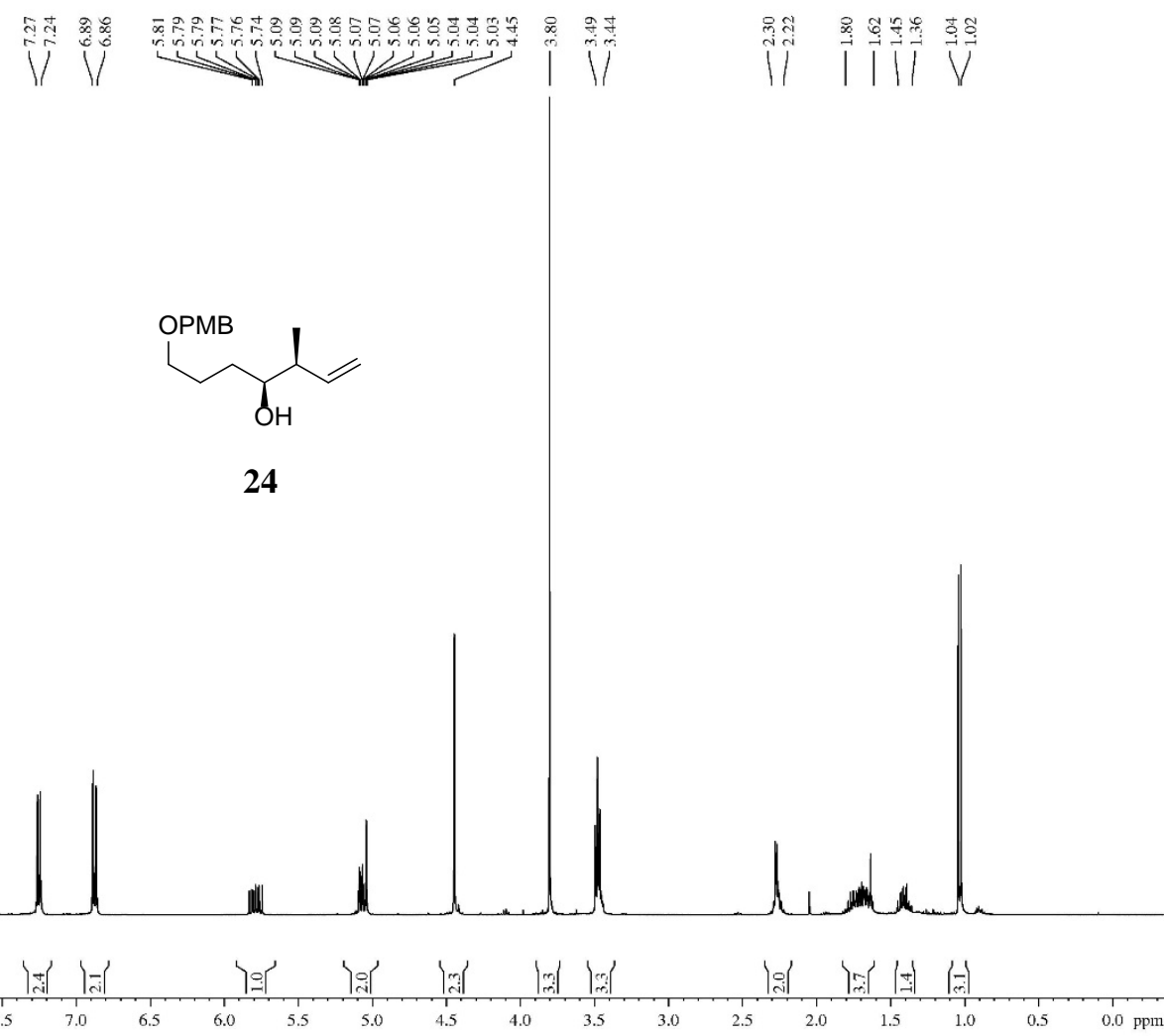

Compound 24, ${ }^{13} \mathrm{C}\left\{{ }^{1} \mathrm{H}\right\}$ NMR (CDCl $3,100 \mathrm{MHz}$ )

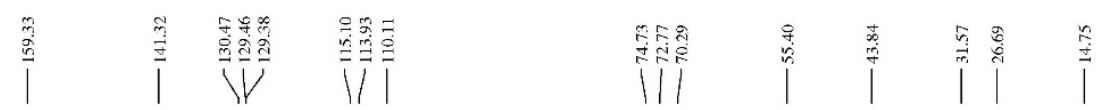

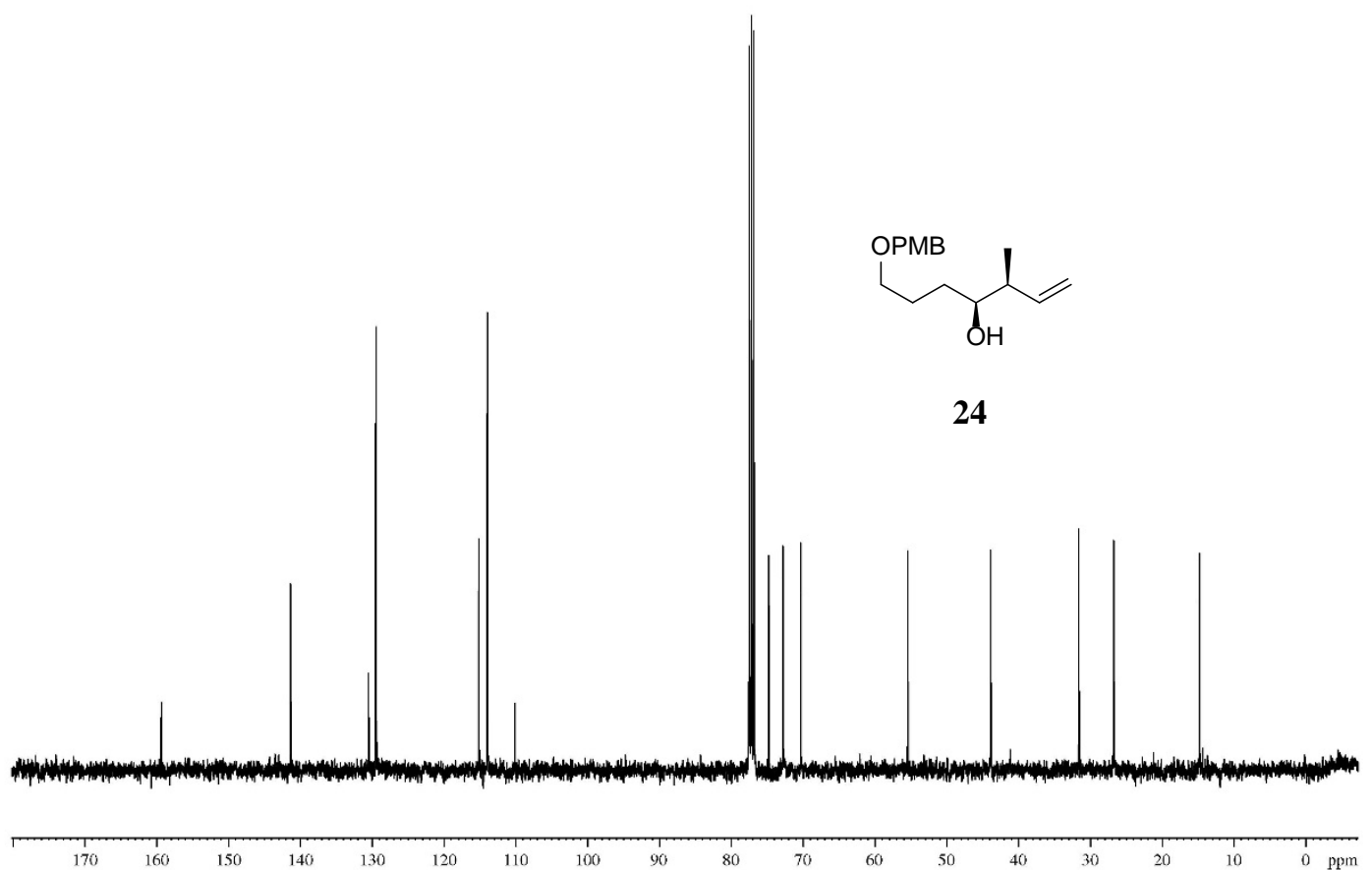


Compound S2, ${ }^{1} \mathrm{H}$ NMR $\left(\mathrm{CDCl}_{3}, 400 \mathrm{MHz}\right)$

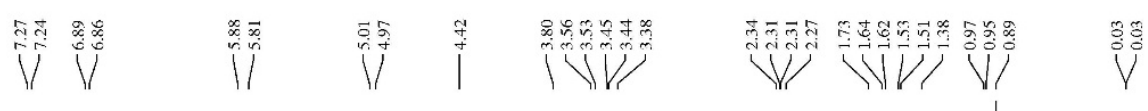<smiles></smiles>

S2
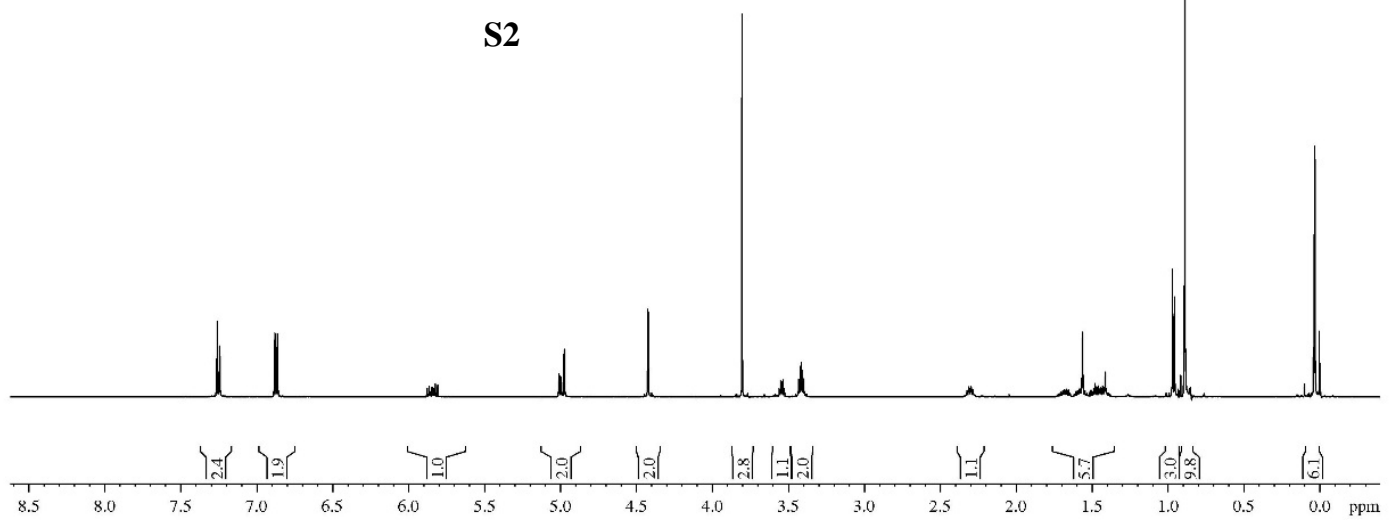

Compound S2, ${ }^{13} \mathrm{C}\left\{{ }^{1} \mathrm{H}\right\} \mathrm{NMR}\left(\mathrm{CDCl}_{3}, 100 \mathrm{MHz}\right)$
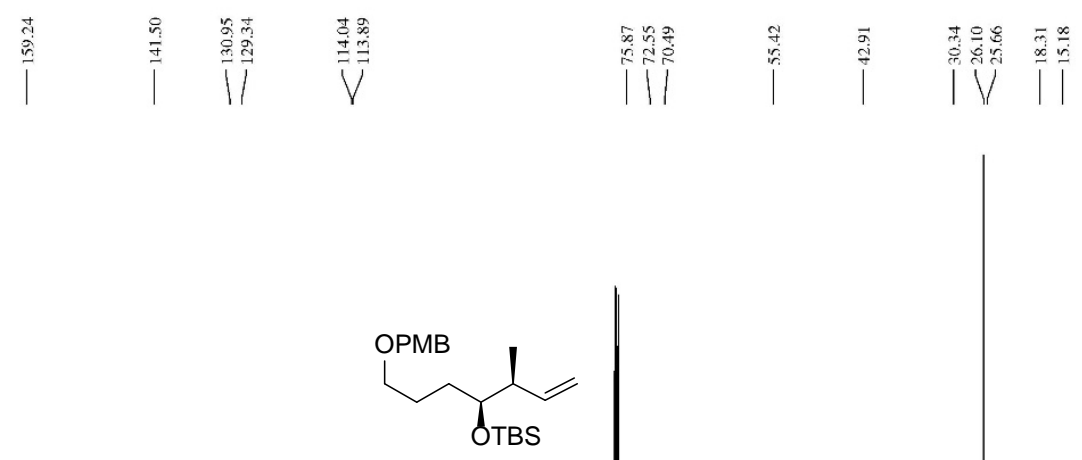

S2

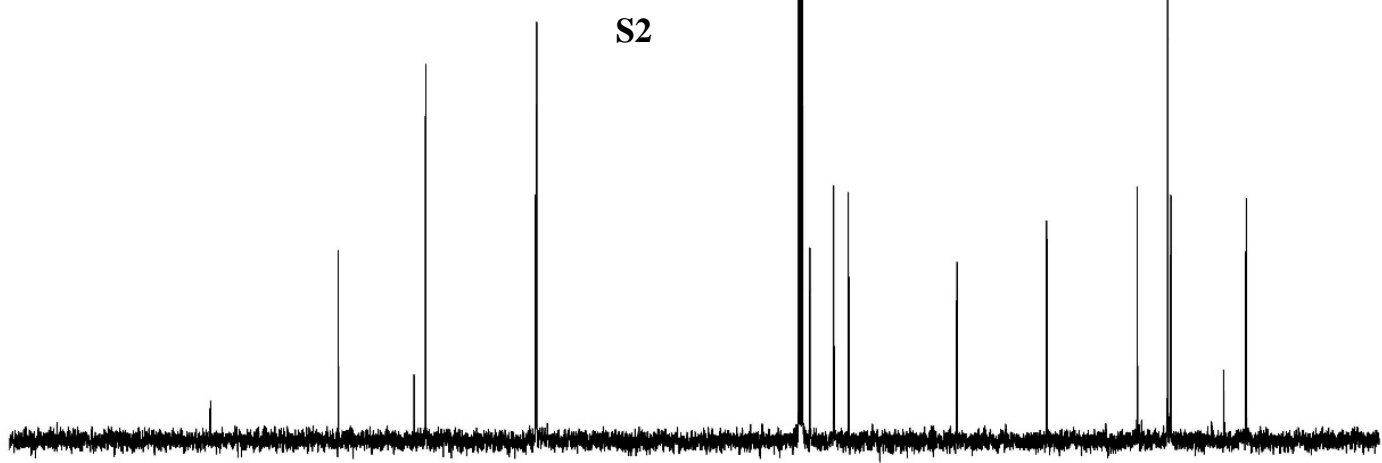

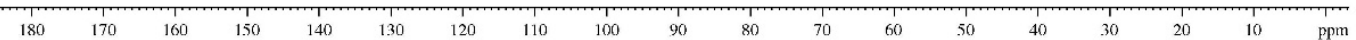


Compound S3, ${ }^{1} \mathrm{H}$ NMR $\left(\mathrm{CDCl}_{3}, 400 \mathrm{MHz}\right)$

$$
\text { 约 }
$$

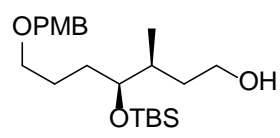

S3
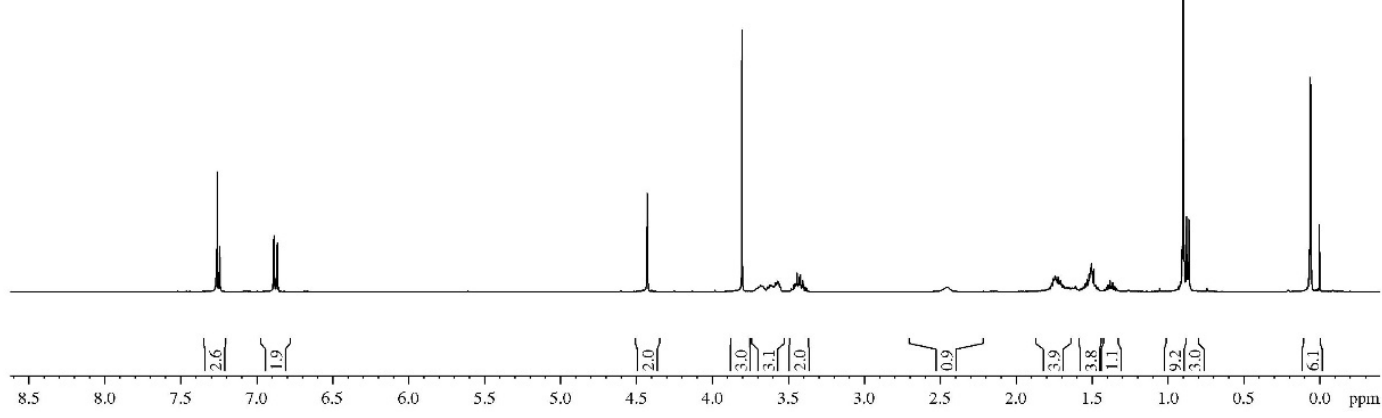

Compound S3, ${ }^{13} \mathrm{C}\left\{{ }^{1} \mathrm{H}\right\}$ NMR $\left(\mathrm{CDCl}_{3}, 100 \mathrm{MHz}\right)$

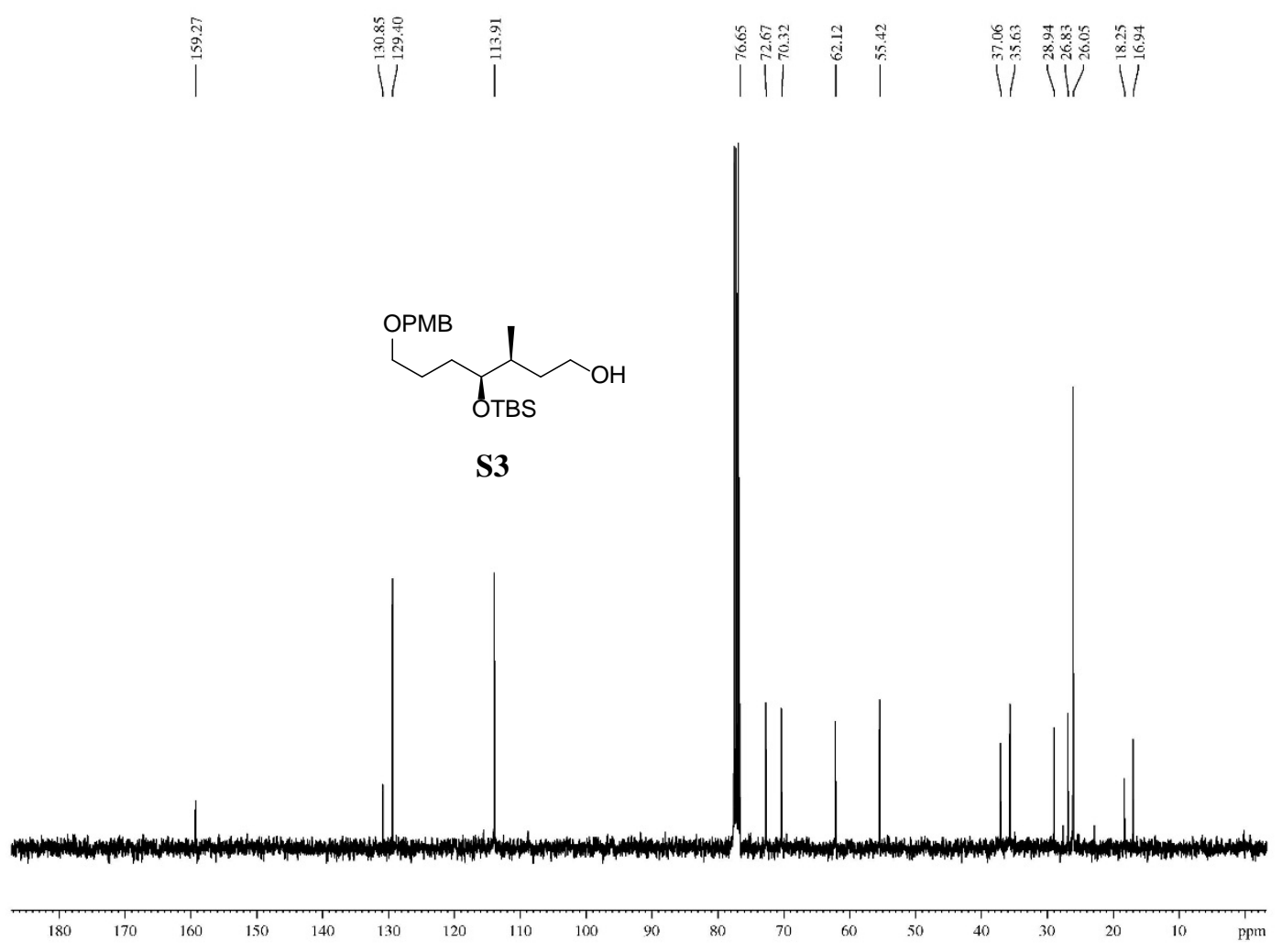


Compound 25, ${ }^{1} \mathrm{H}$ NMR $\left(\mathrm{CDCl}_{3}, 400 \mathrm{MHz}\right)$
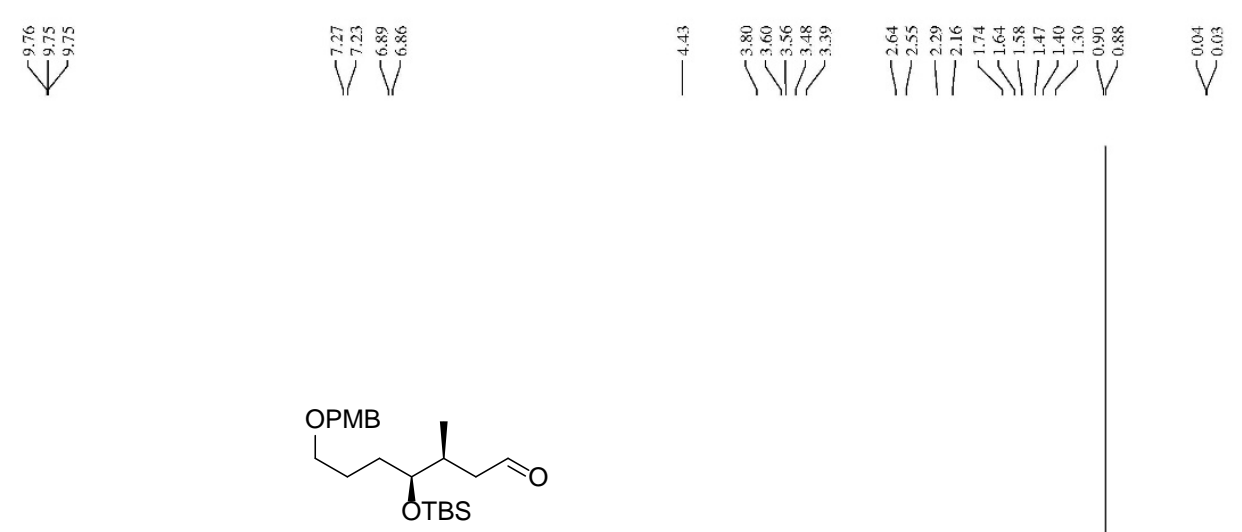

25

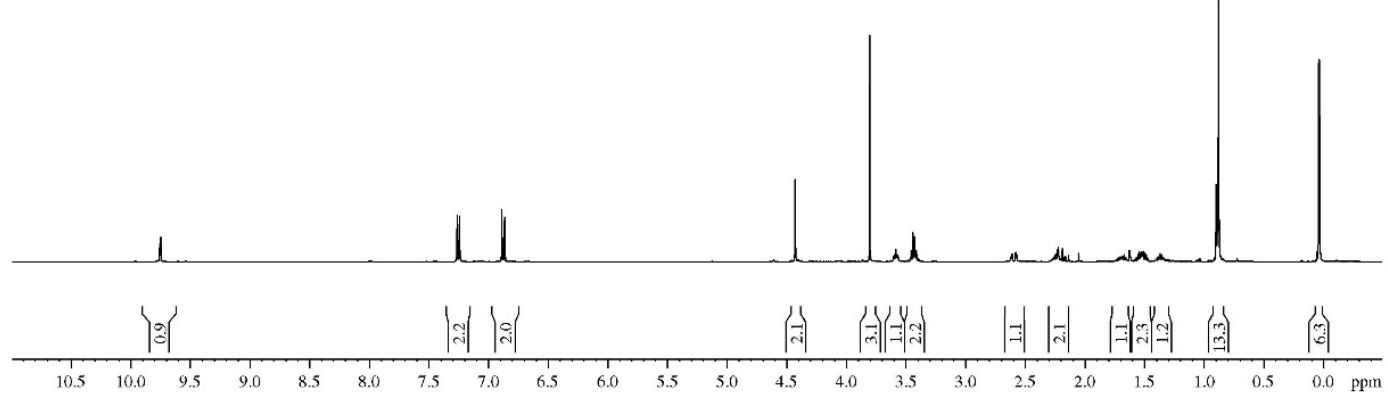

Compound 25, ${ }^{13} \mathrm{C}\left\{{ }^{1} \mathrm{H}\right\}$ NMR $\left(\mathrm{CDCl}_{3}, 100 \mathrm{MHz}\right)$
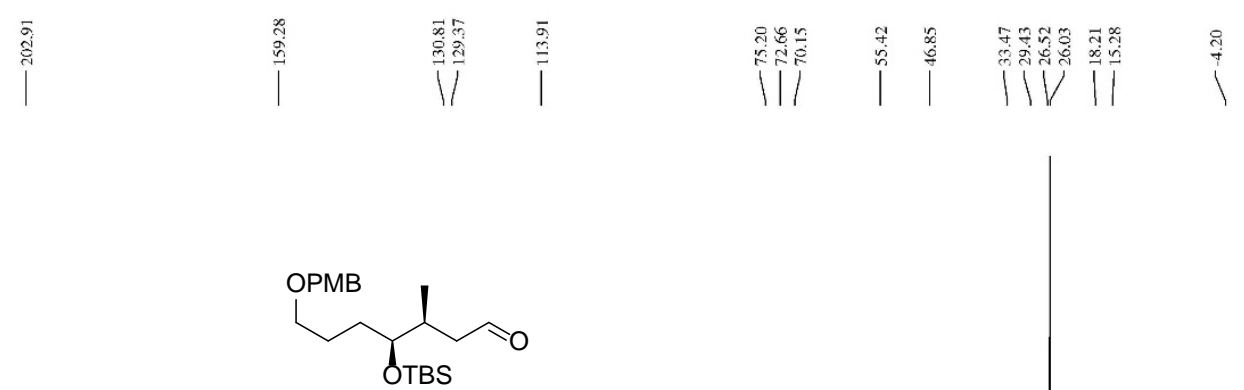

25

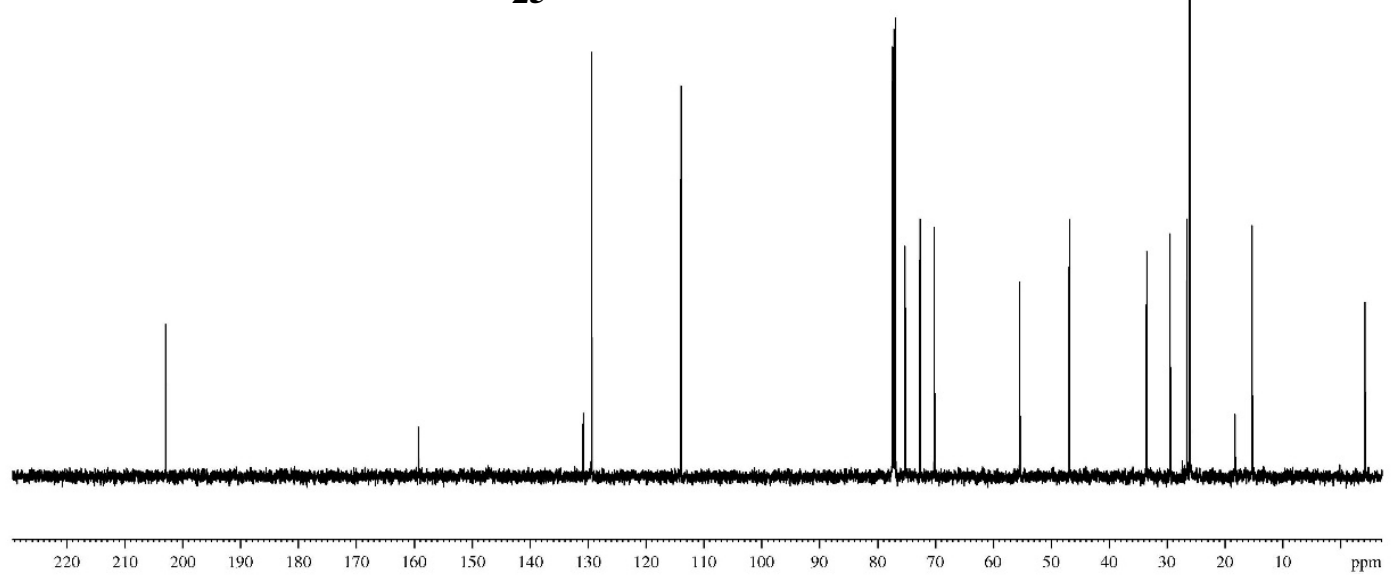


Compound 26, $\left(\mathrm{CDCl}_{3}, 400 \mathrm{MHz}\right)$

$\prod^{1}$
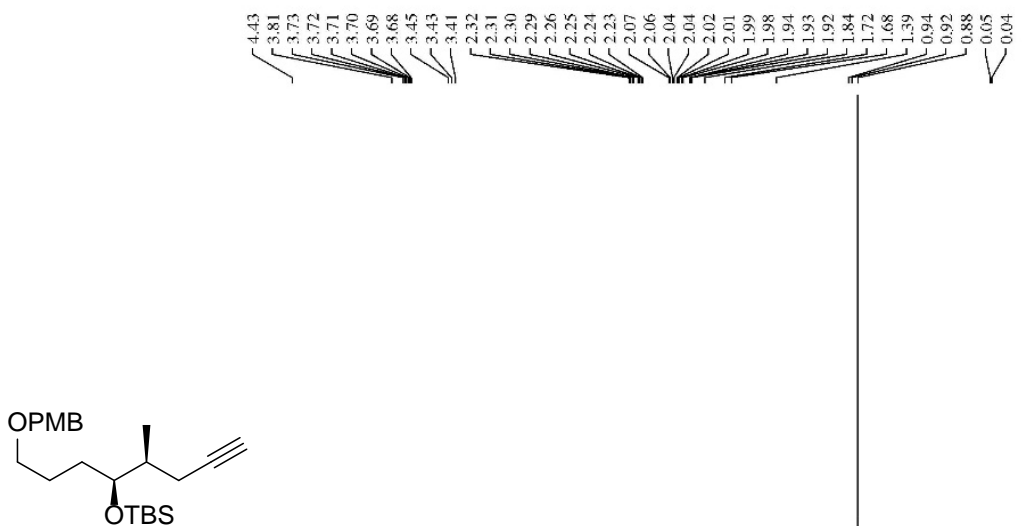

26
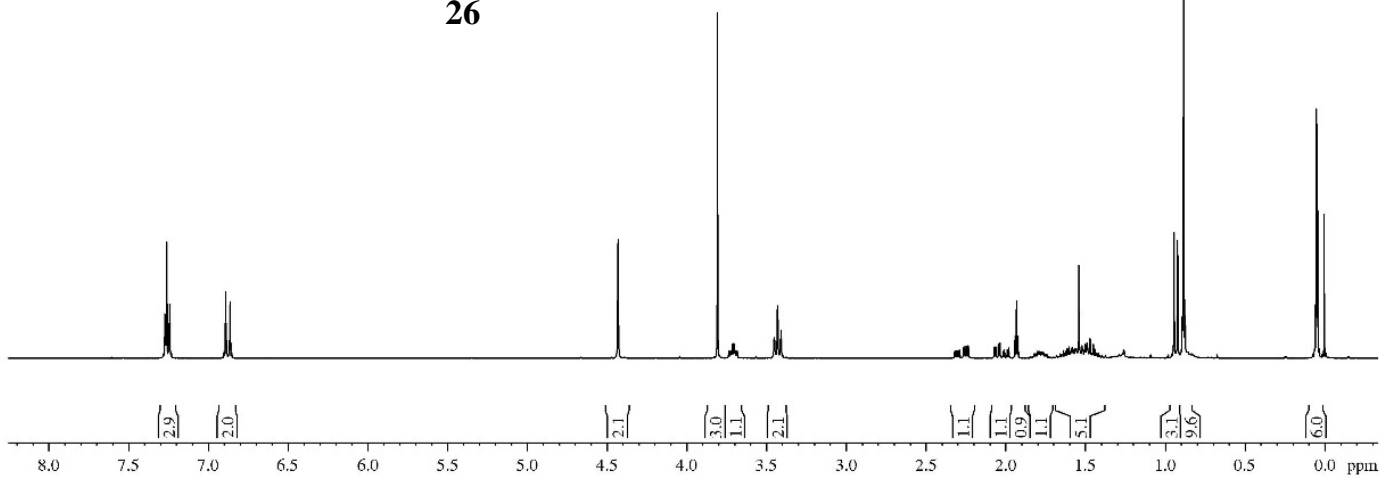

Compound 26, ${ }^{13} \mathrm{C}\left\{{ }^{1} \mathrm{H}\right\}$ NMR $\left(\mathrm{CDCl}_{3}, 100 \mathrm{MHz}\right)$

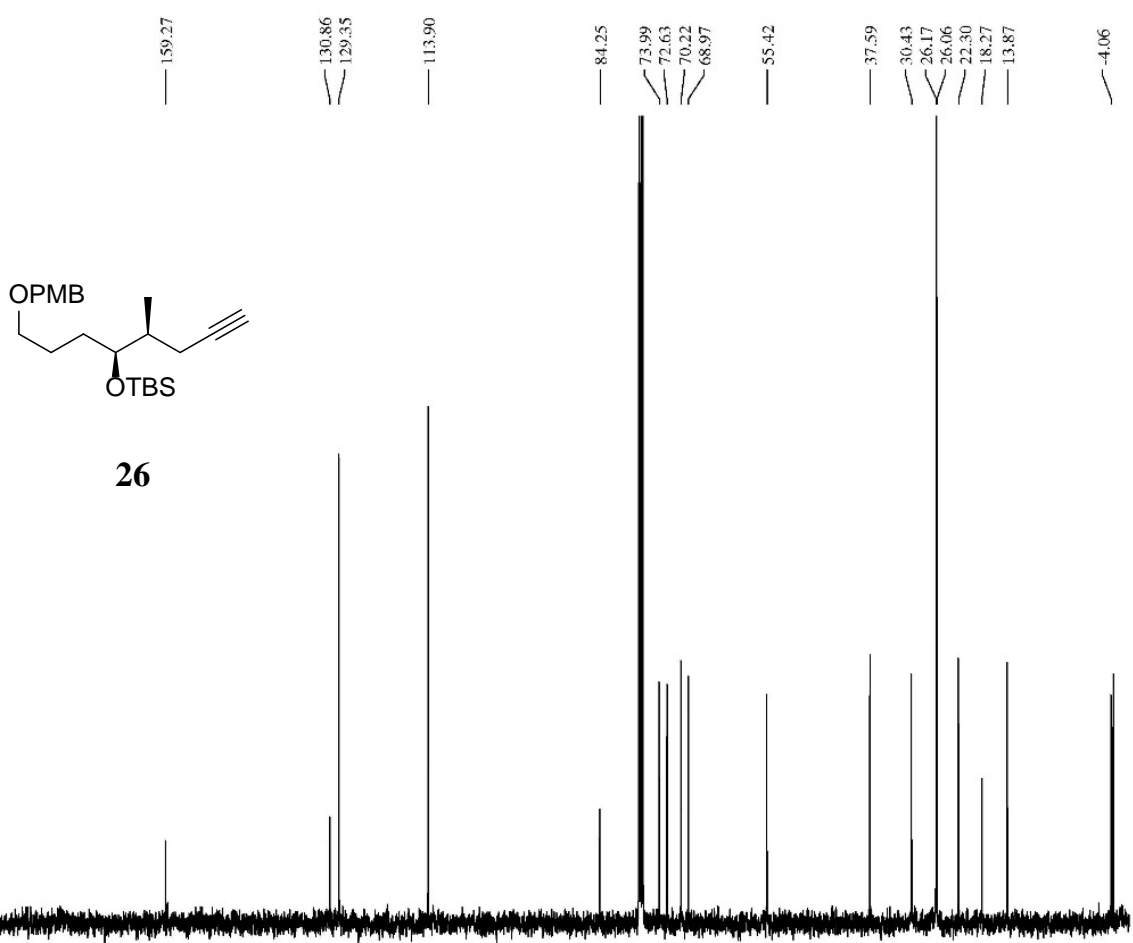

$\begin{array}{llllllllllllllllllllll}220 & 210 & 200 & 190 & 180 & 170 & 160 & 150 & 140 & 130 & 120 & 110 & 100 & 90 & 80 & 70 & 60 & 50 & 40 & 30 & 20 & 10\end{array}$ 
Compound S4, ${ }^{1} \mathrm{H}$ NMR $\left(\mathrm{CDCl}_{3}, 400 \mathrm{MHz}\right)$

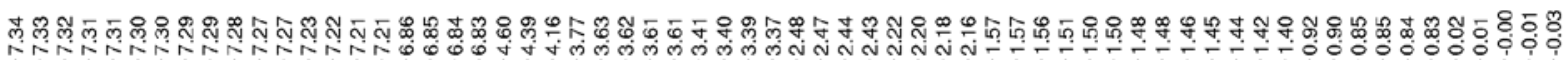
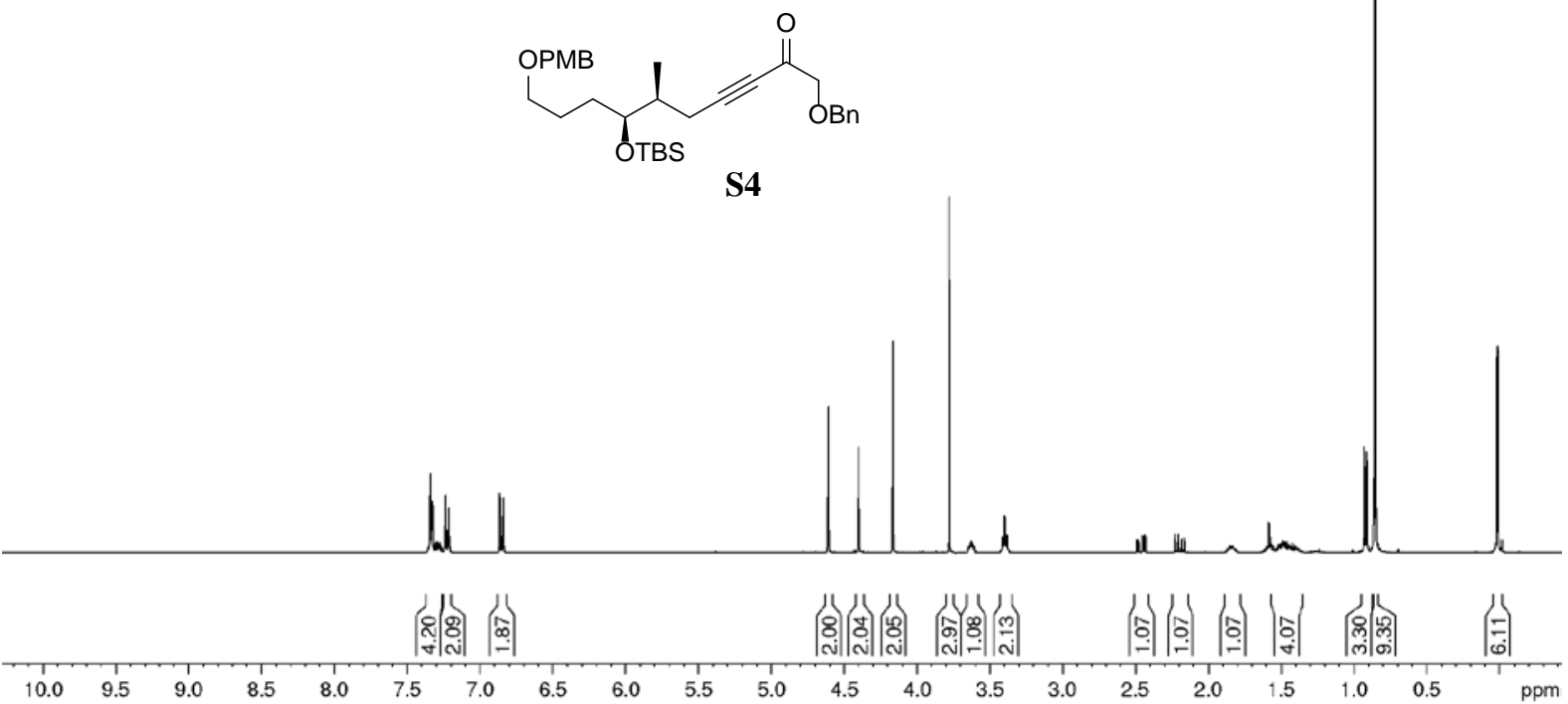

Compound S4, ${ }^{13} \mathrm{C}\left\{{ }^{1} \mathrm{H}\right\}\left(\mathrm{CDCl}_{3}, 100 \mathrm{MHz}\right)$

| |

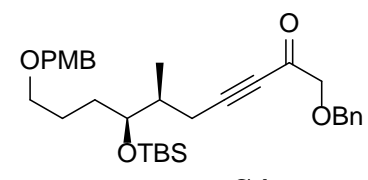

S4

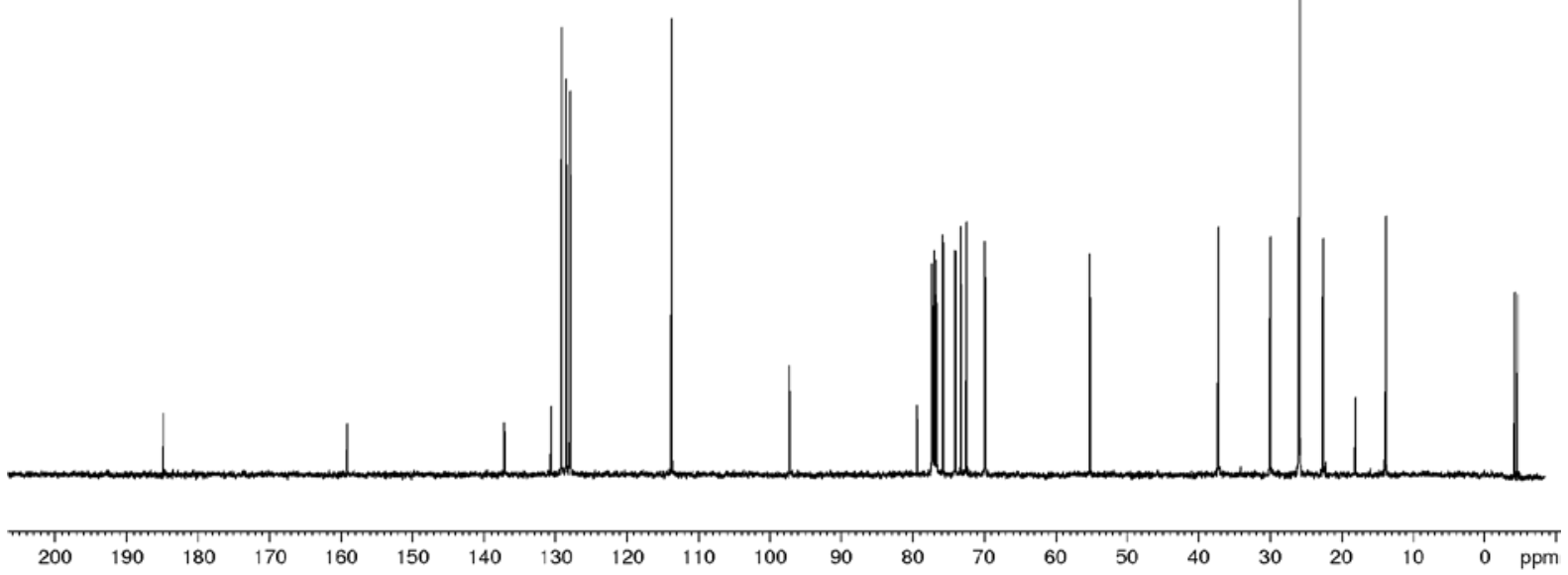




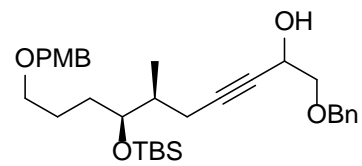

28

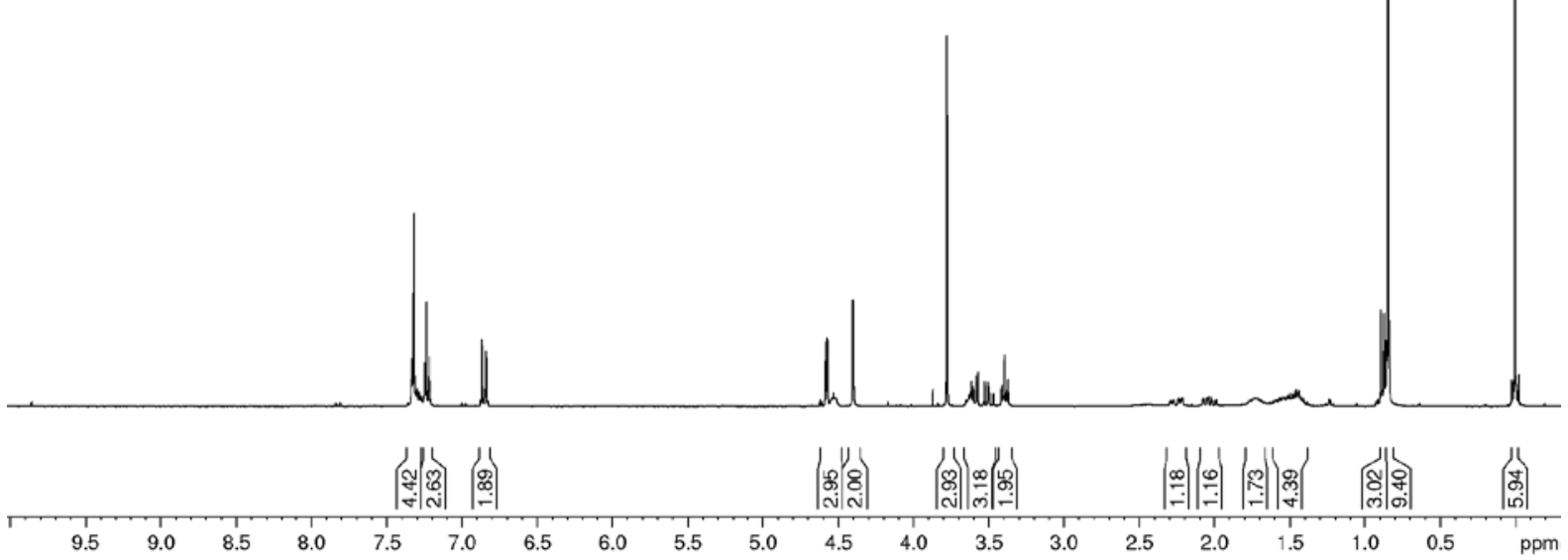

Compound 28, ${ }^{13} \mathrm{C}\left\{{ }^{1} \mathrm{H}\right\}\left(\mathrm{CDCl}_{3}, 100 \mathrm{MHz}\right)$
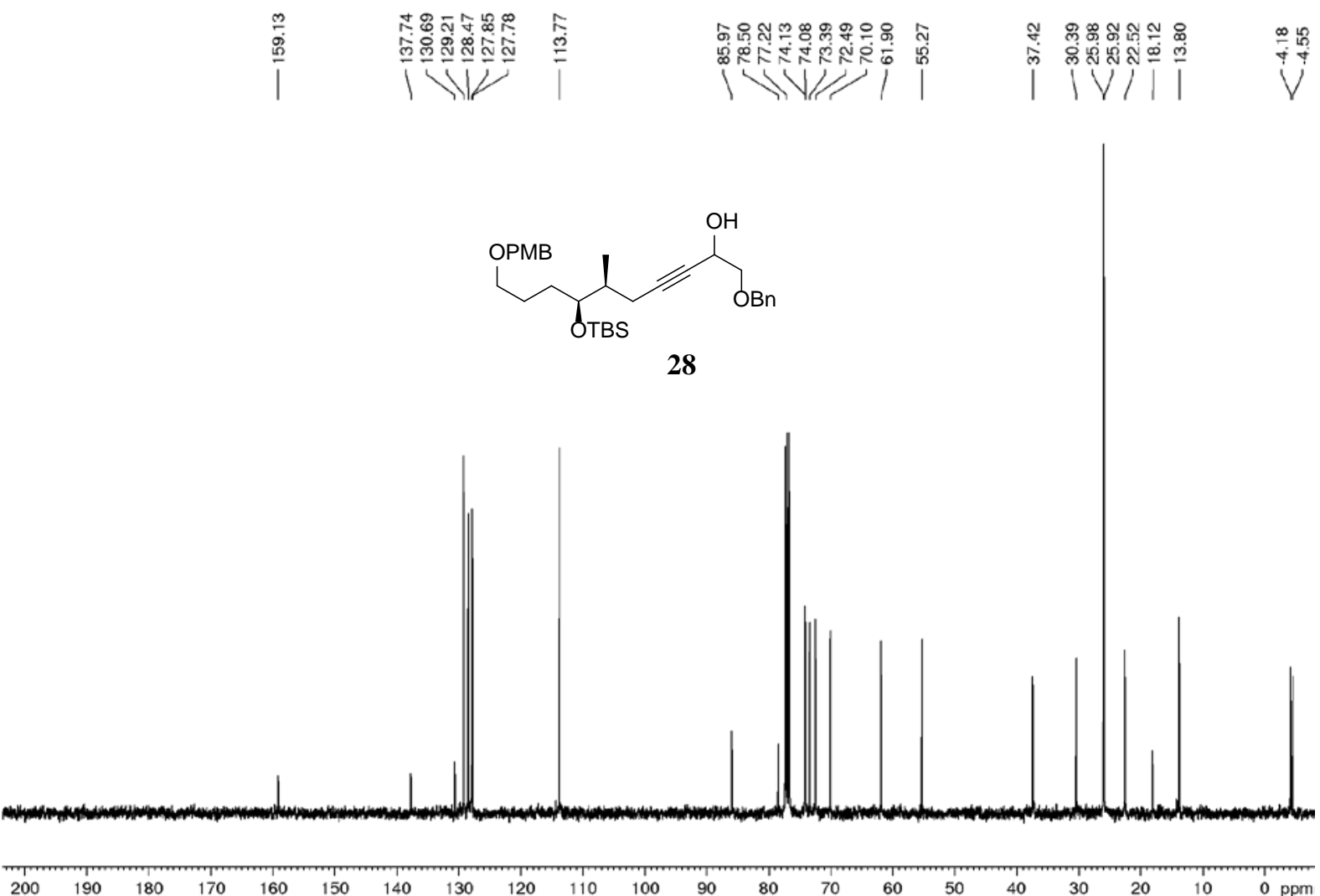


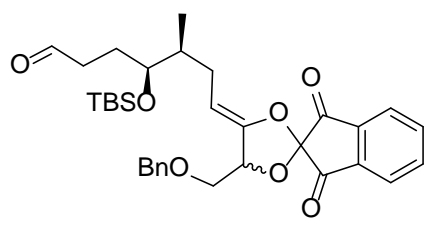

29

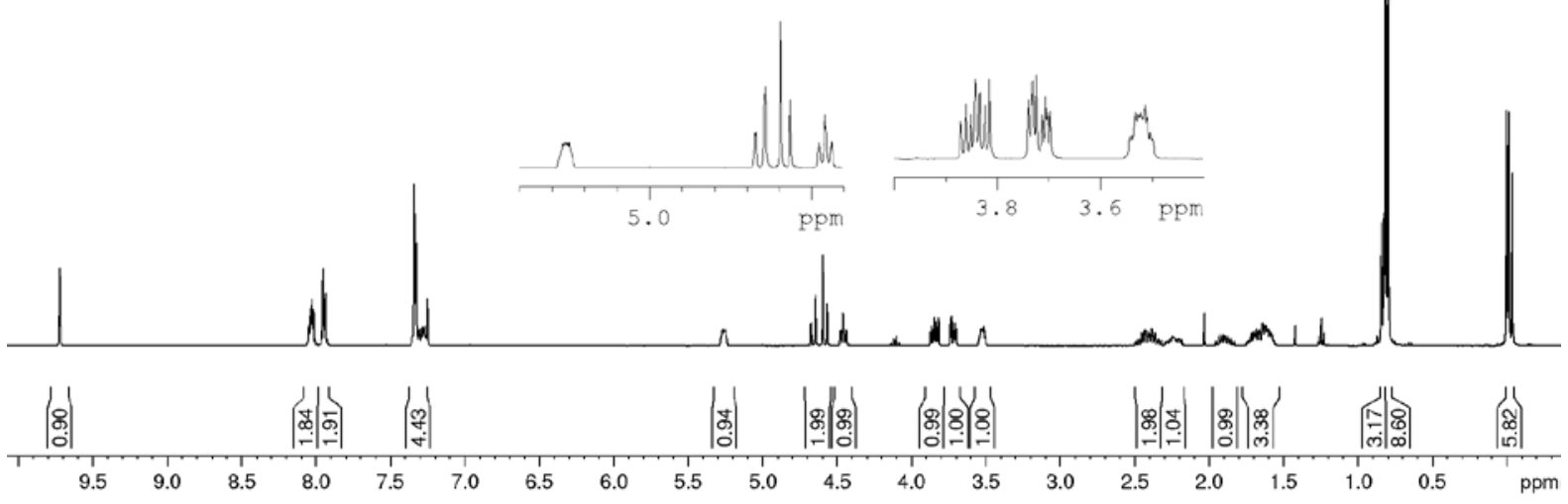

Compound 29, ${ }^{13} \mathrm{C}\left\{{ }^{1} \mathrm{H}\right\}\left(\mathrm{CDCl}_{3}, 100 \mathrm{MHz}\right)$
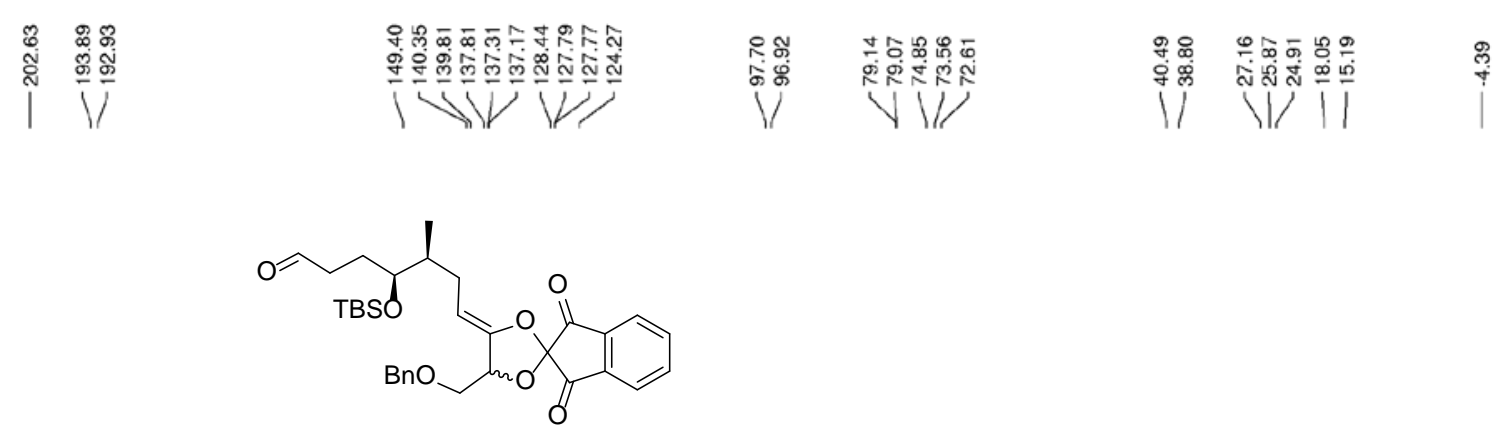

29

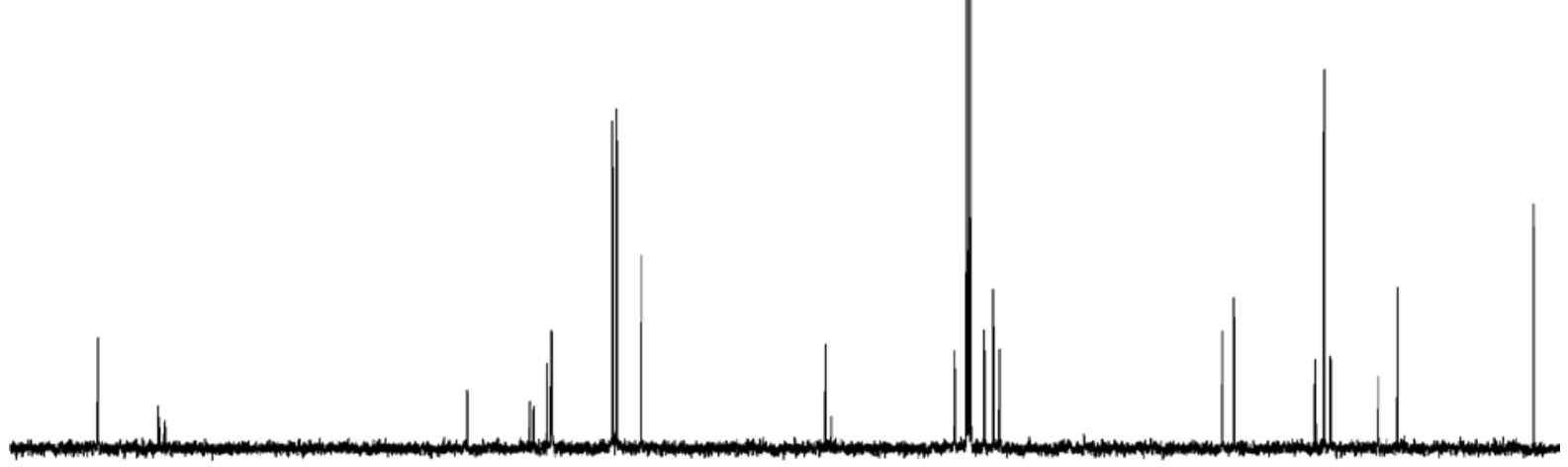

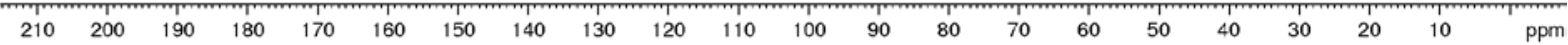


Compound 31, ${ }^{1} \mathrm{H}$ NMR $\left(\mathrm{CDCl}_{3}, 400 \mathrm{MHz}\right)$
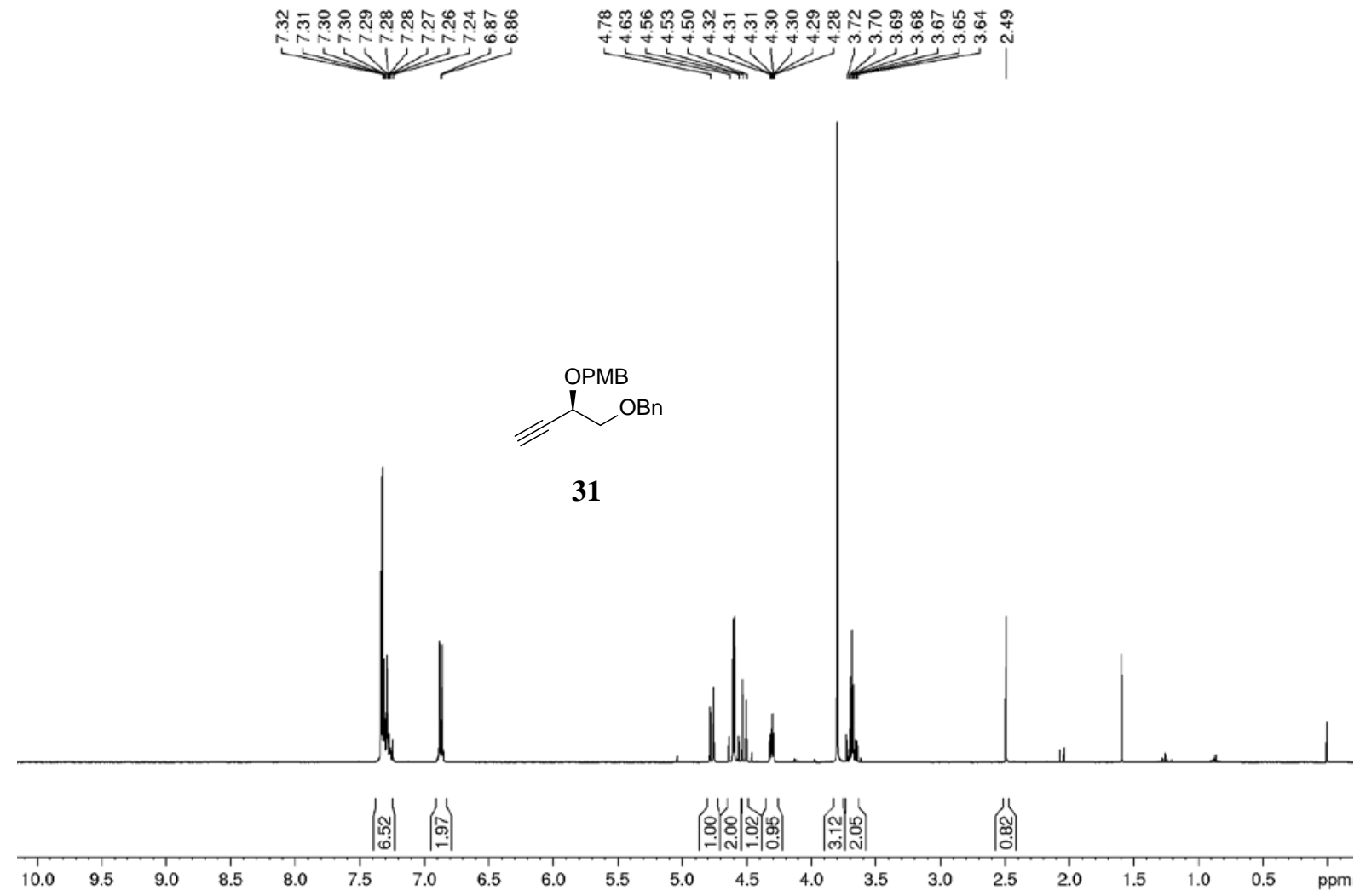

Compound 31, ${ }^{13} \mathrm{C}\left\{{ }^{1} \mathrm{H}\right\}\left(\mathrm{CDCl}_{3}, 100 \mathrm{MHz}\right)$
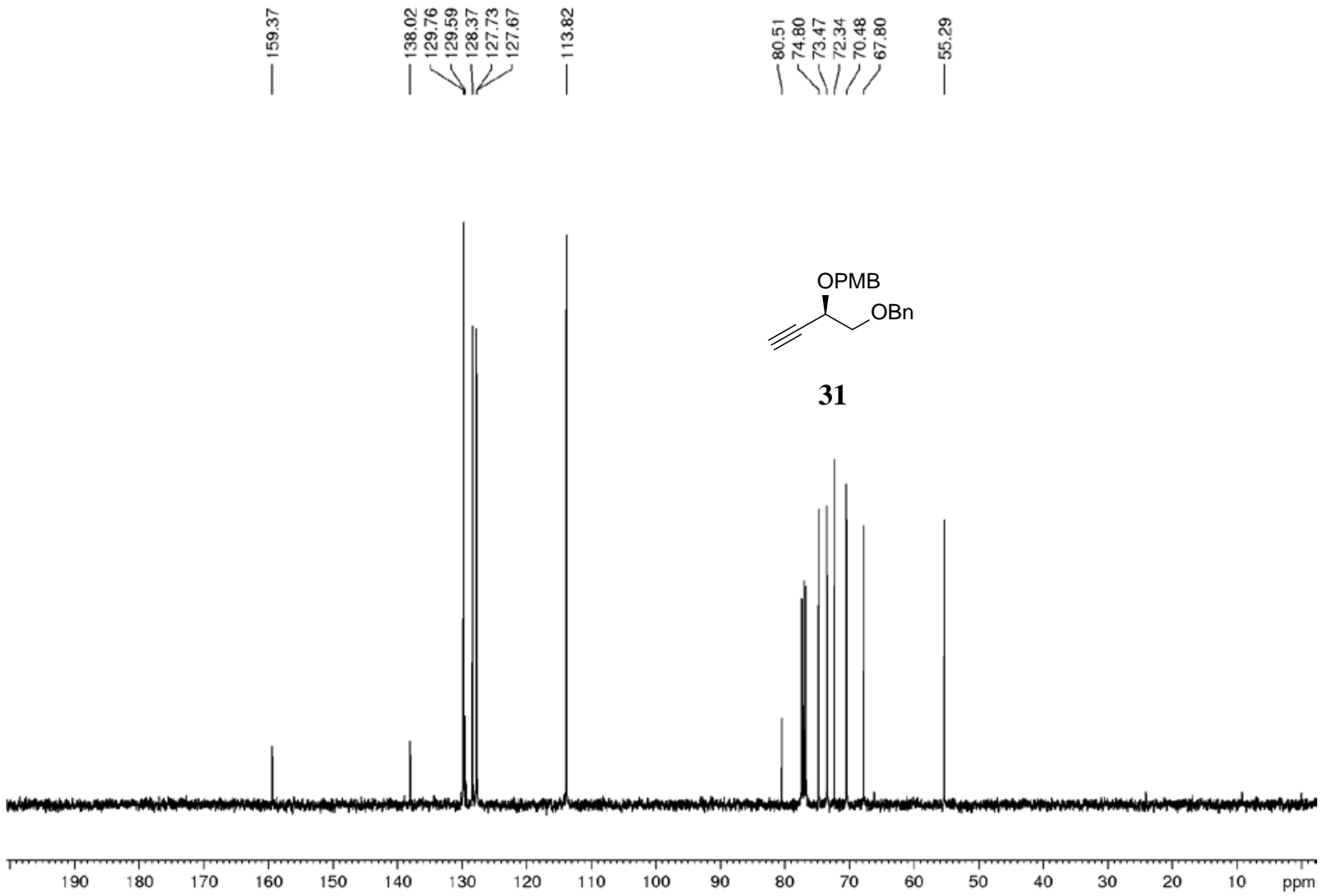

年

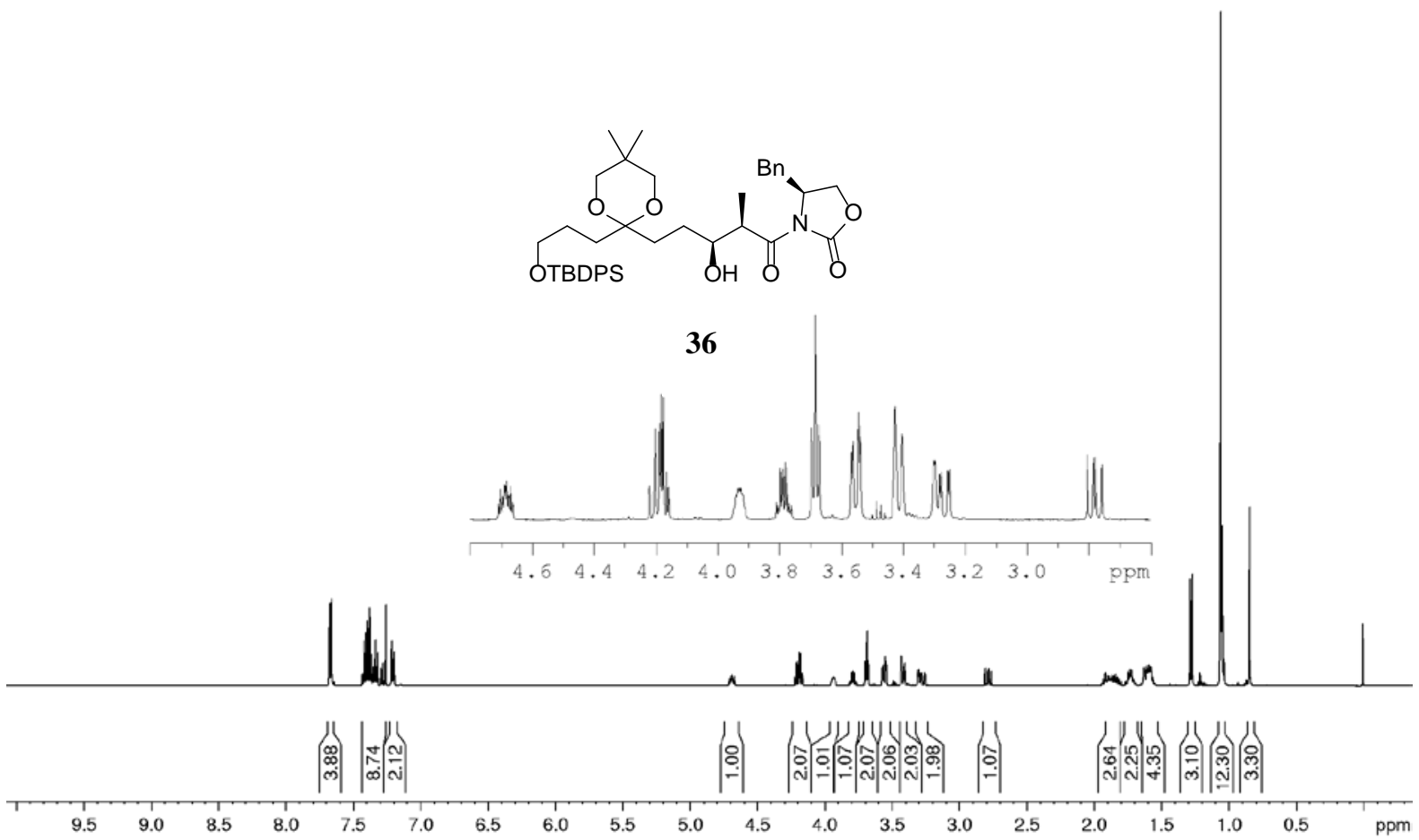

Compound 36, ${ }^{13} \mathrm{C}\left\{{ }^{1} \mathrm{H}\right\}$ NMR $\left(\mathrm{CDCl}_{3}, 100 \mathrm{MHz}\right)$

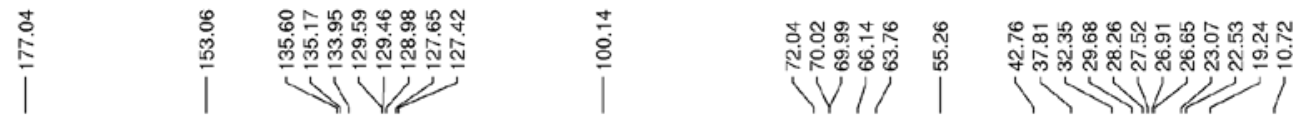

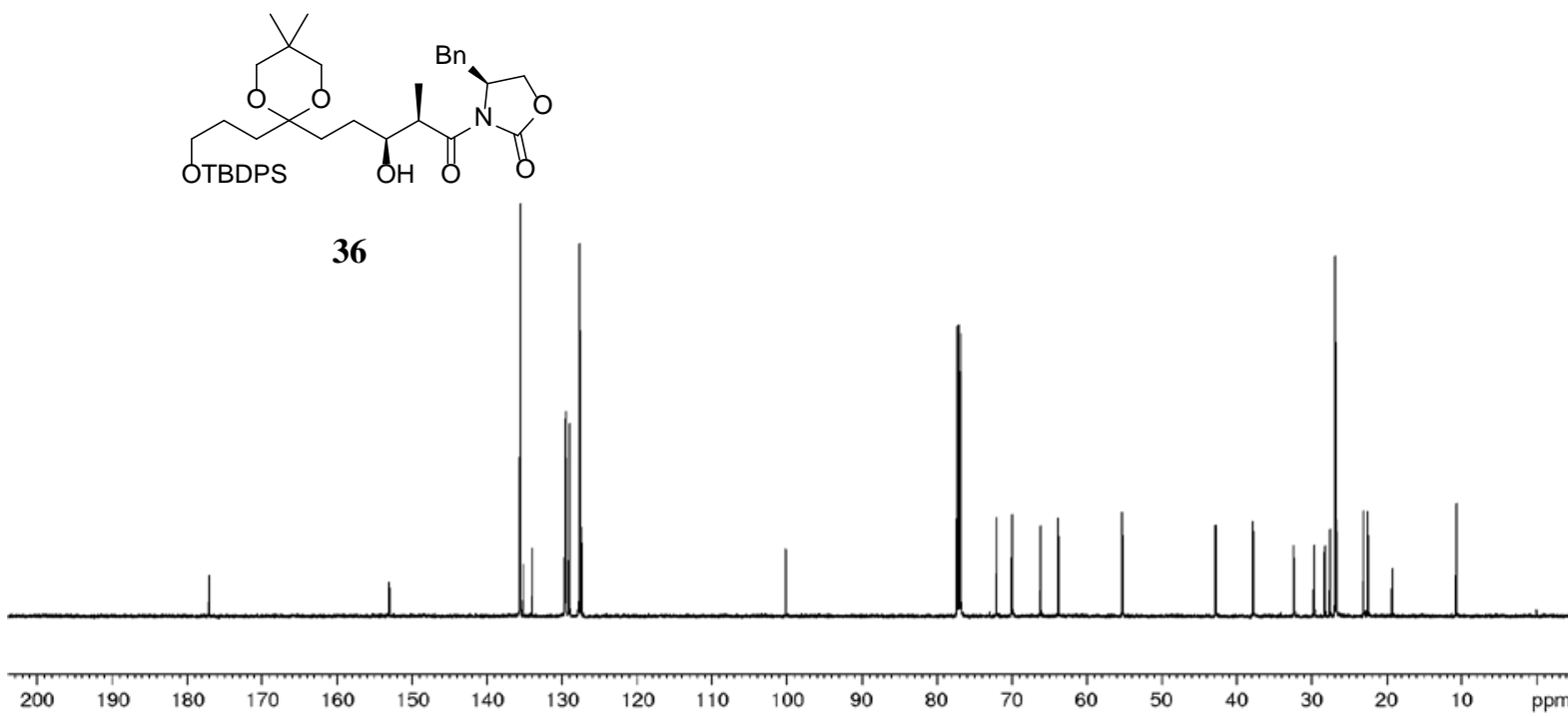




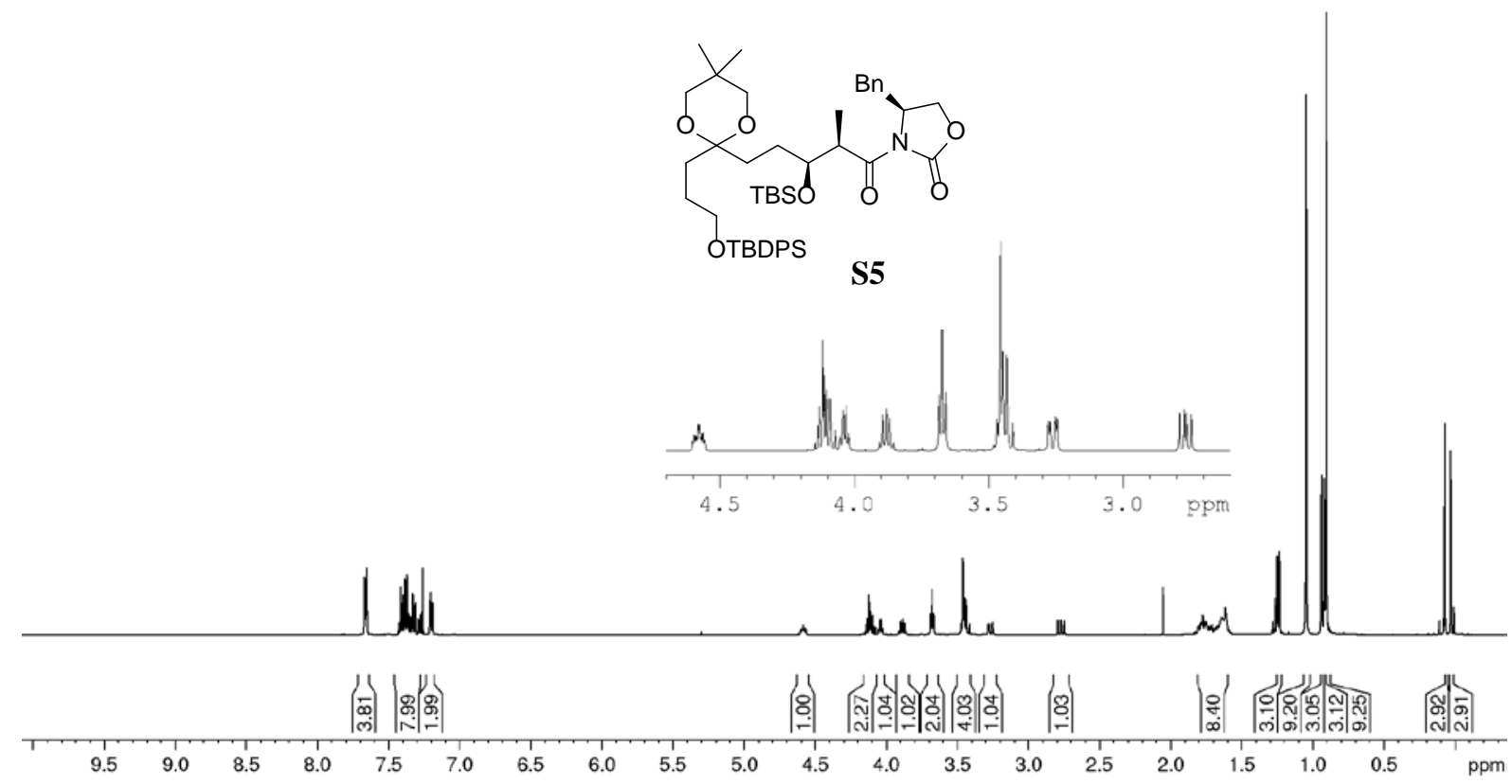

Compound S5, ${ }^{13} \mathrm{C}\left\{{ }^{1} \mathrm{H}\right\}\left(\mathrm{CDCl}_{3}, 100 \mathrm{MHz}\right)$
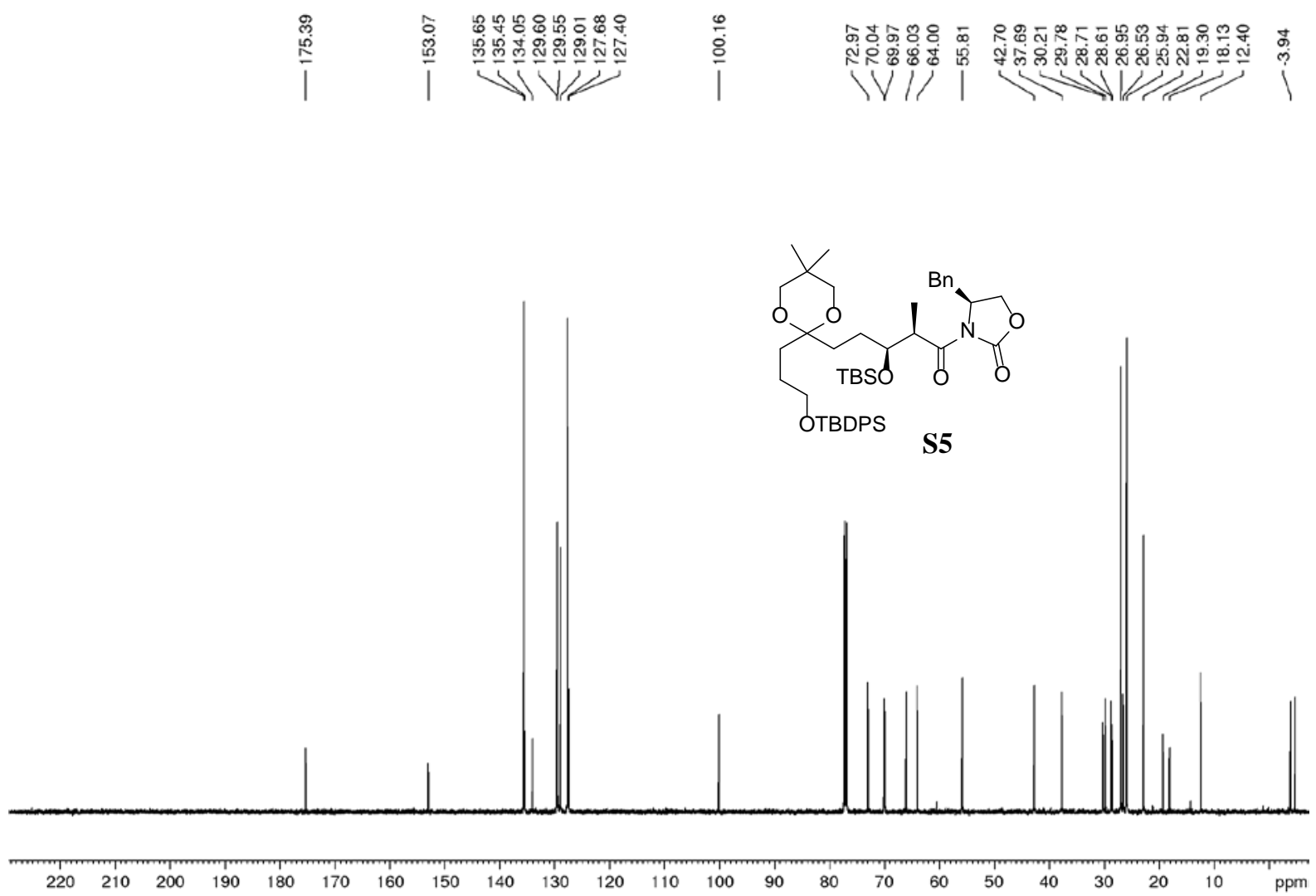
<smiles>CC(CO)C(C)CCC1(CCCO[18O])OCC(C)(C)CO1</smiles>

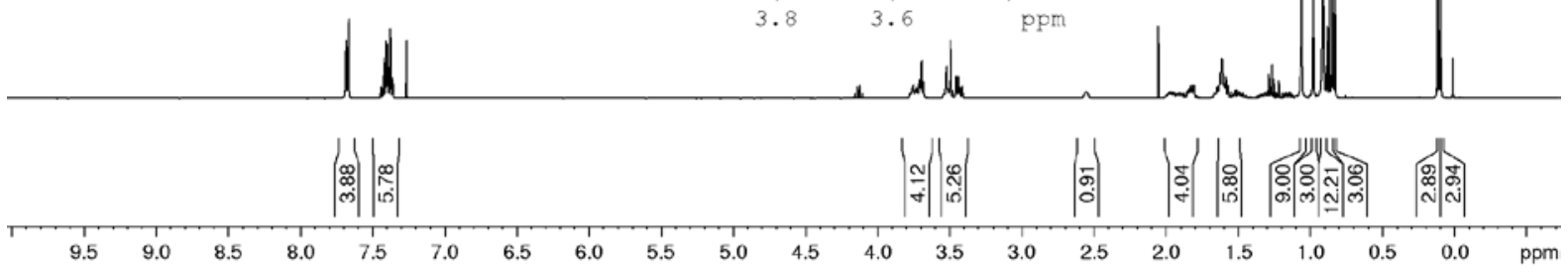

Compound 37, ${ }^{13} \mathrm{C}\left\{{ }^{1} \mathrm{H}\right\}\left(\mathrm{CDCl}_{3}, 100 \mathrm{MHz}\right)$

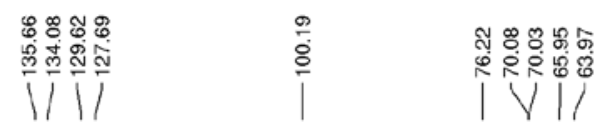

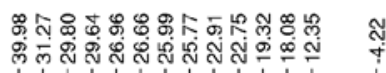

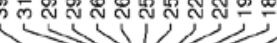

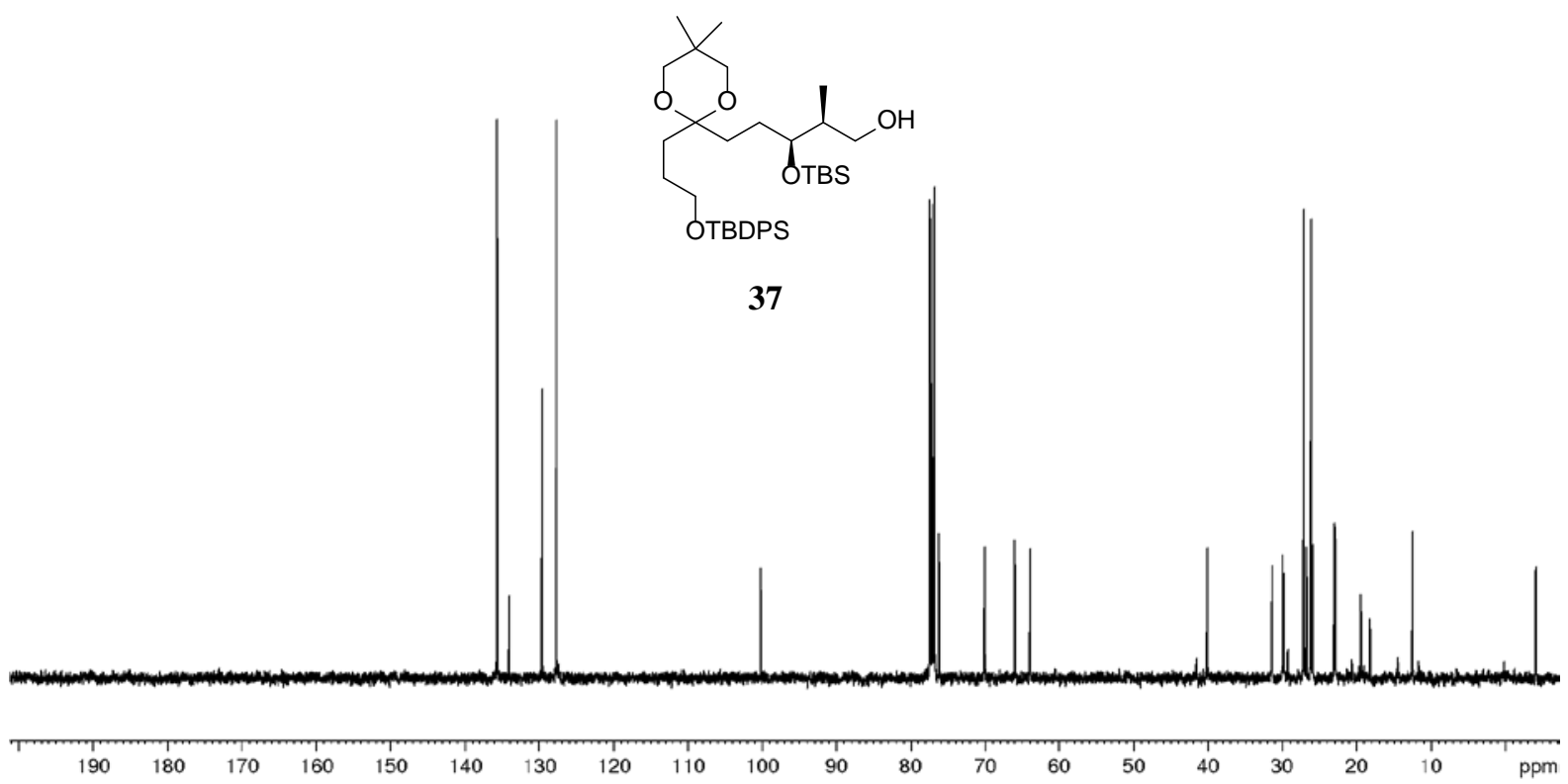



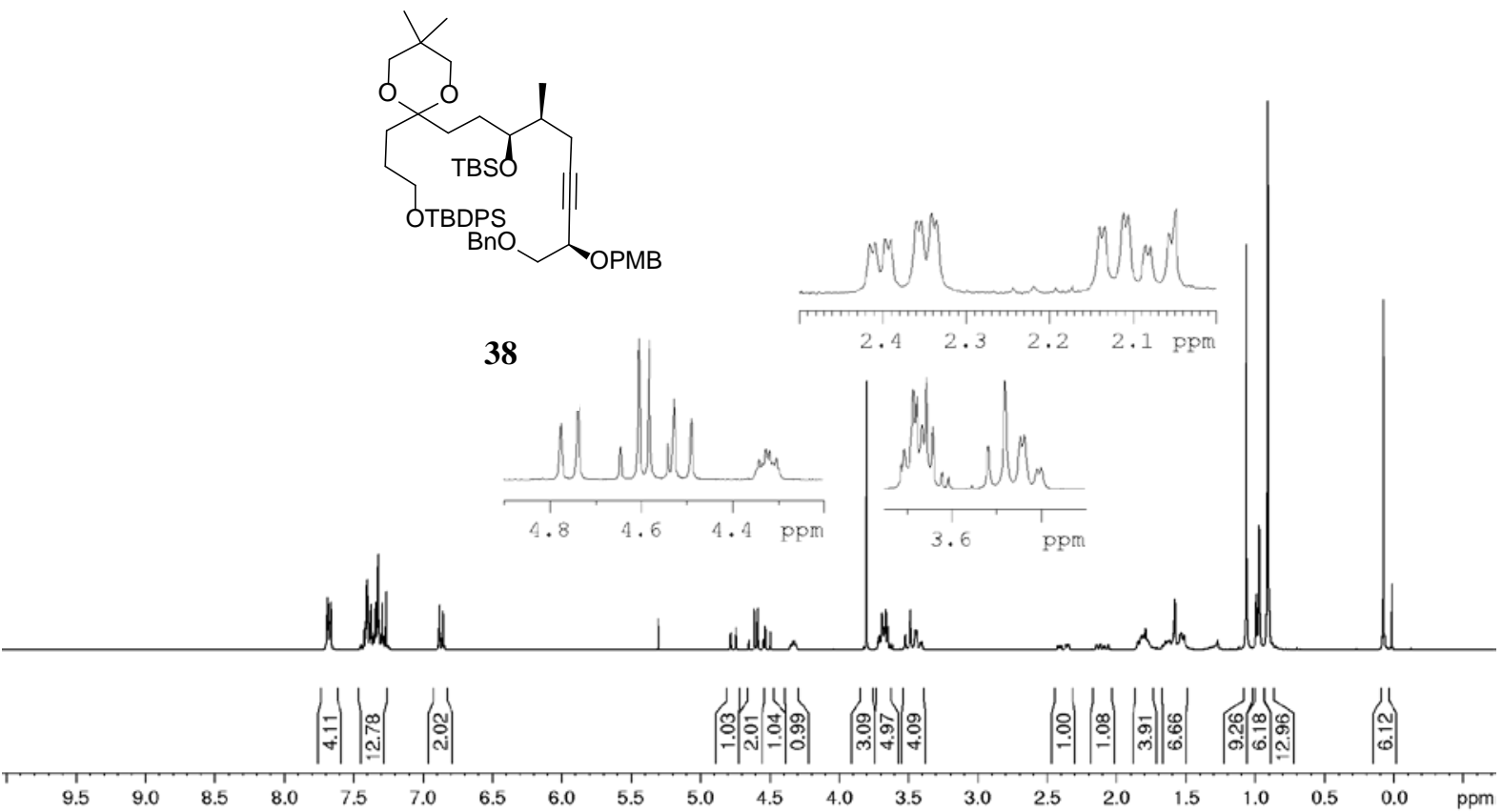

38
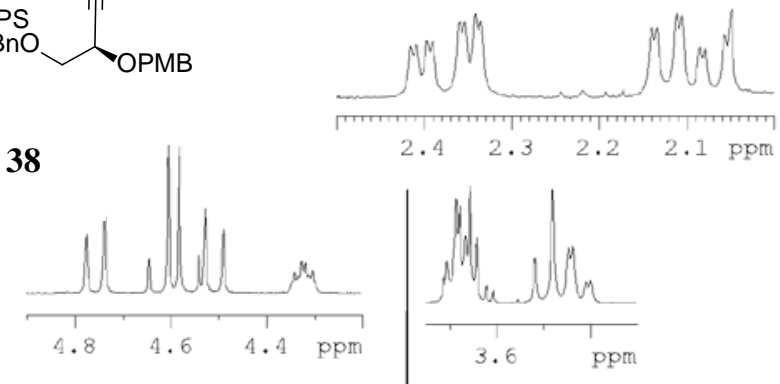

Compound 38, ${ }^{13} \mathrm{C}\left\{{ }^{1} \mathrm{H}\right\}\left(\mathrm{CDCl}_{3}, 100 \mathrm{MHz}\right)$

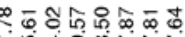

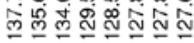

1)

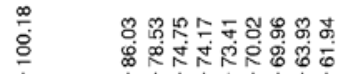

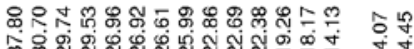
(र)

Nañ

$+N$

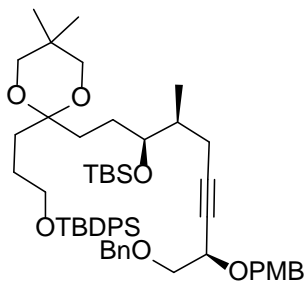

38

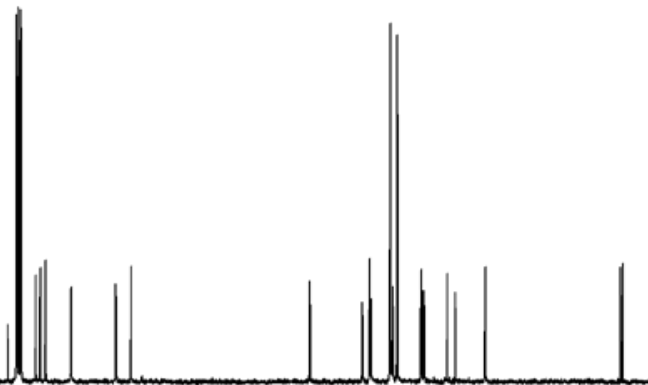




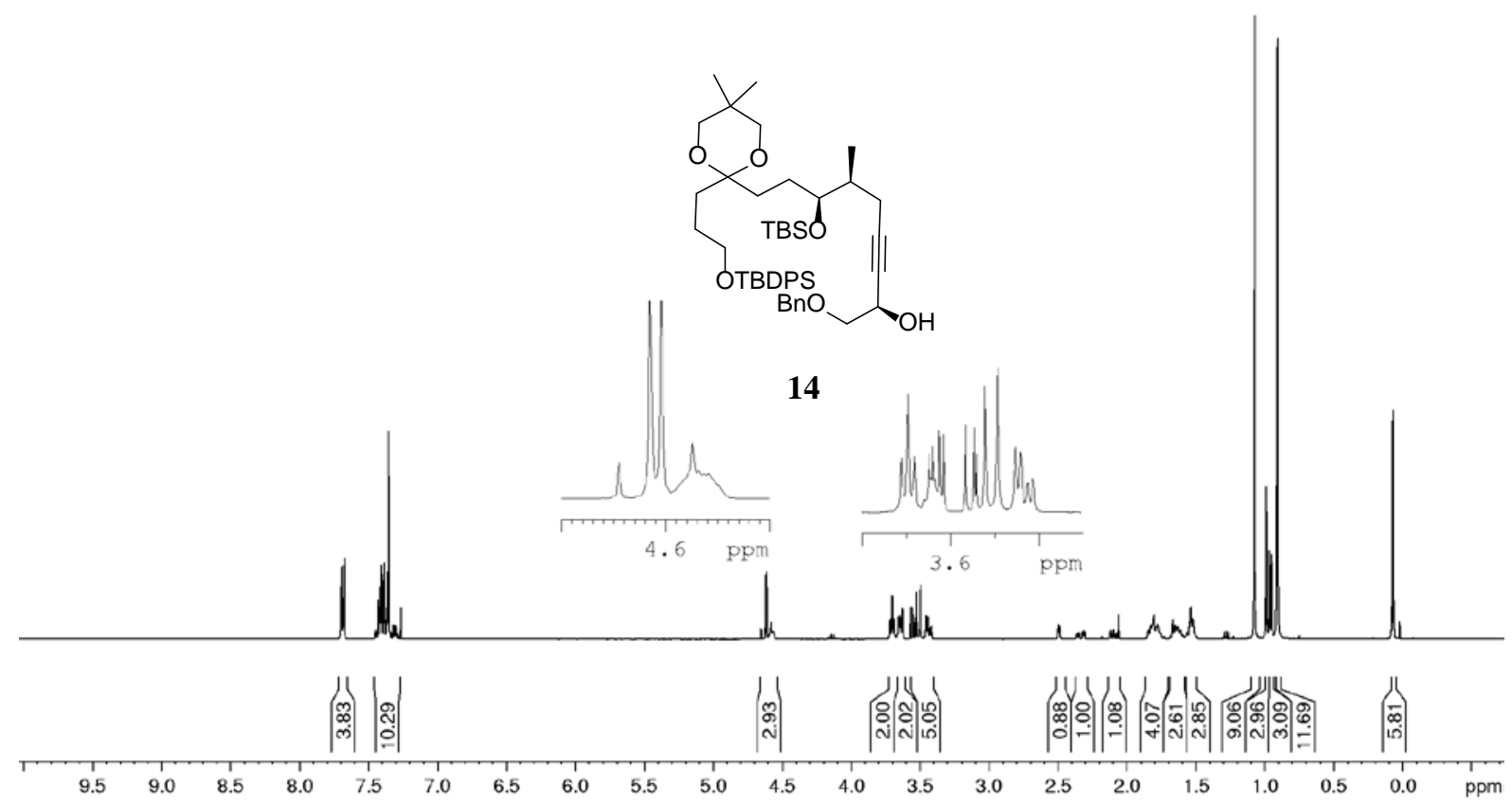

Compound 14, ${ }^{13} \mathrm{C}\left\{{ }^{1} \mathrm{H}\right\}\left(\mathrm{CDCl}_{3}, 100 \mathrm{MHz}\right)$

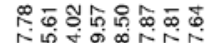

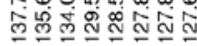
)

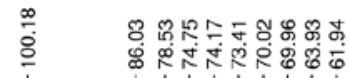

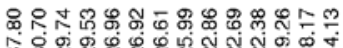

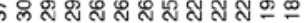

(1)
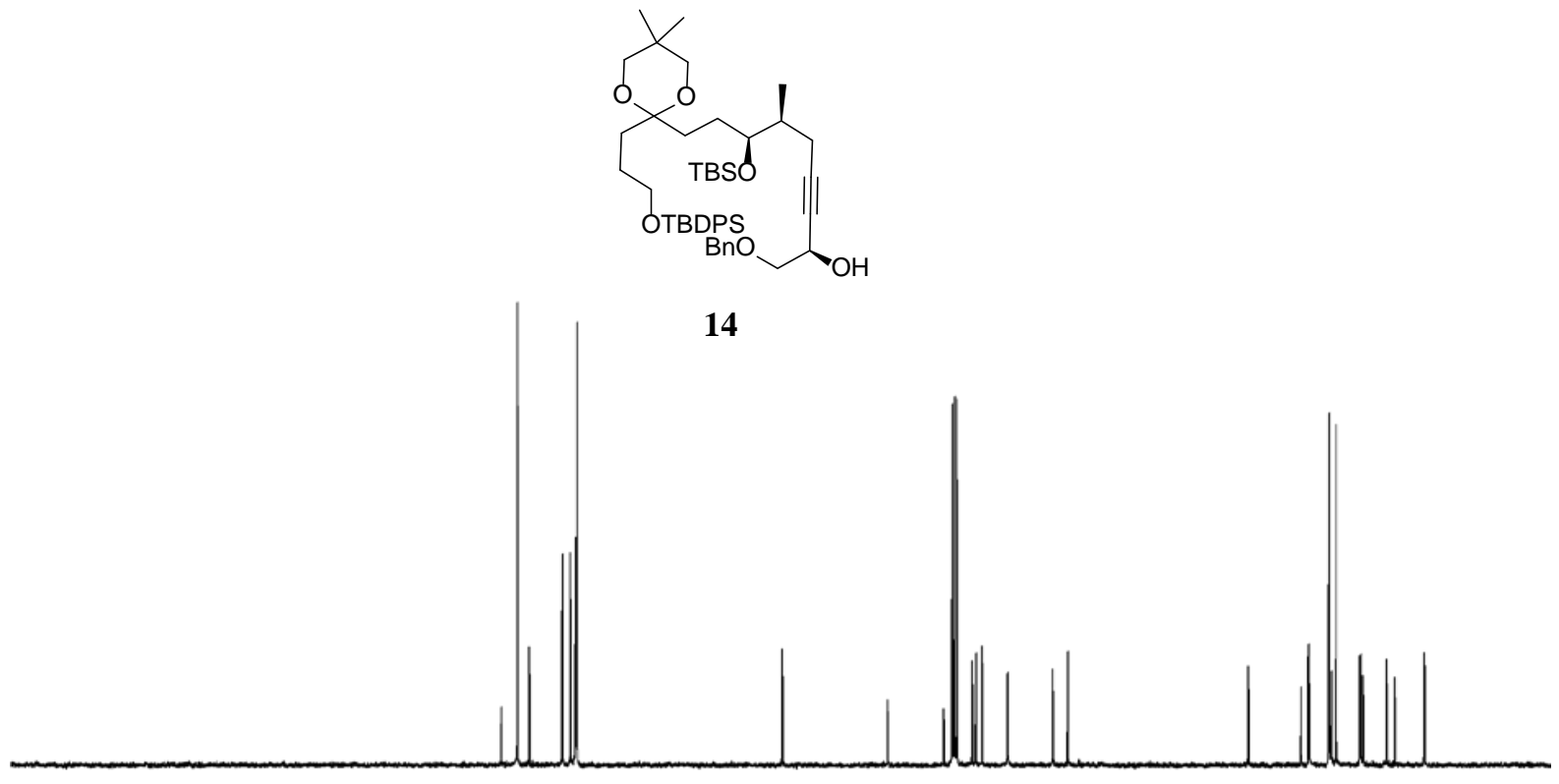

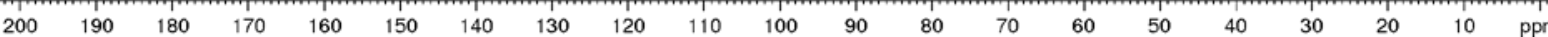


Compound 13, ${ }^{1} \mathrm{H} \mathrm{NMR}\left(\mathrm{CDCl}_{3}, 400 \mathrm{MHz}\right)$ 若象

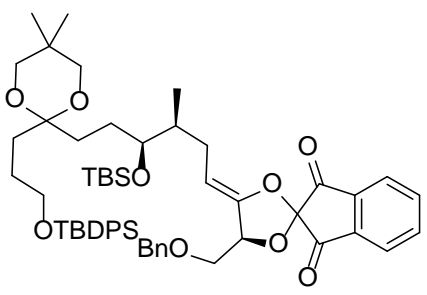

13

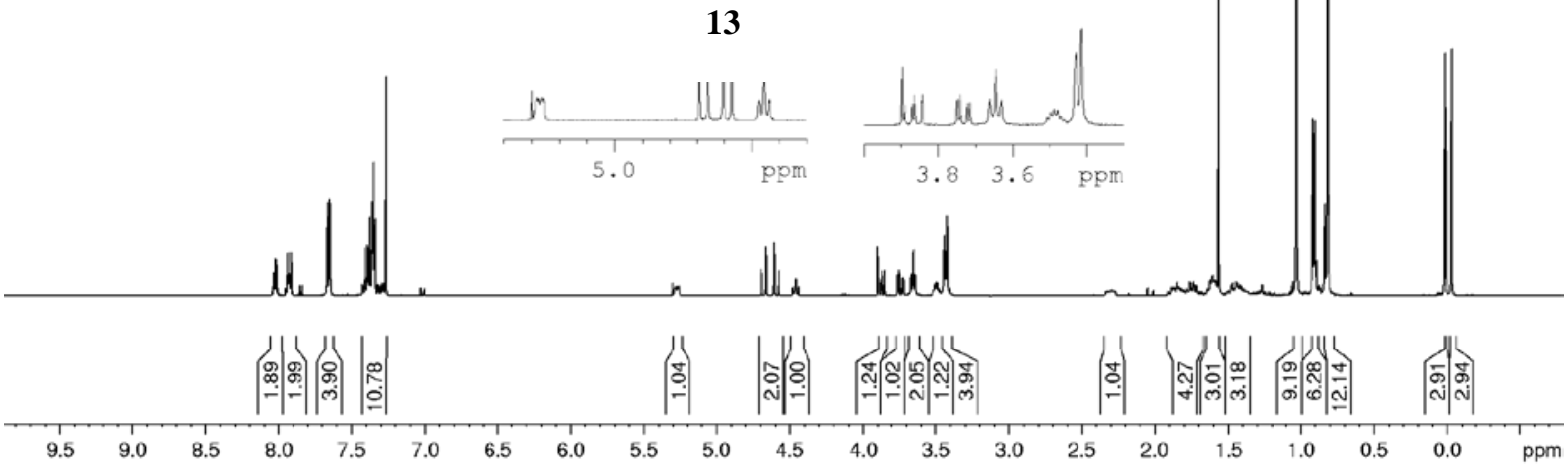

Compound 13, ${ }^{13} \mathrm{C}\left\{{ }^{1} \mathrm{H}\right\}\left(\mathrm{CDCl}_{3}, 100 \mathrm{MHz}\right)$
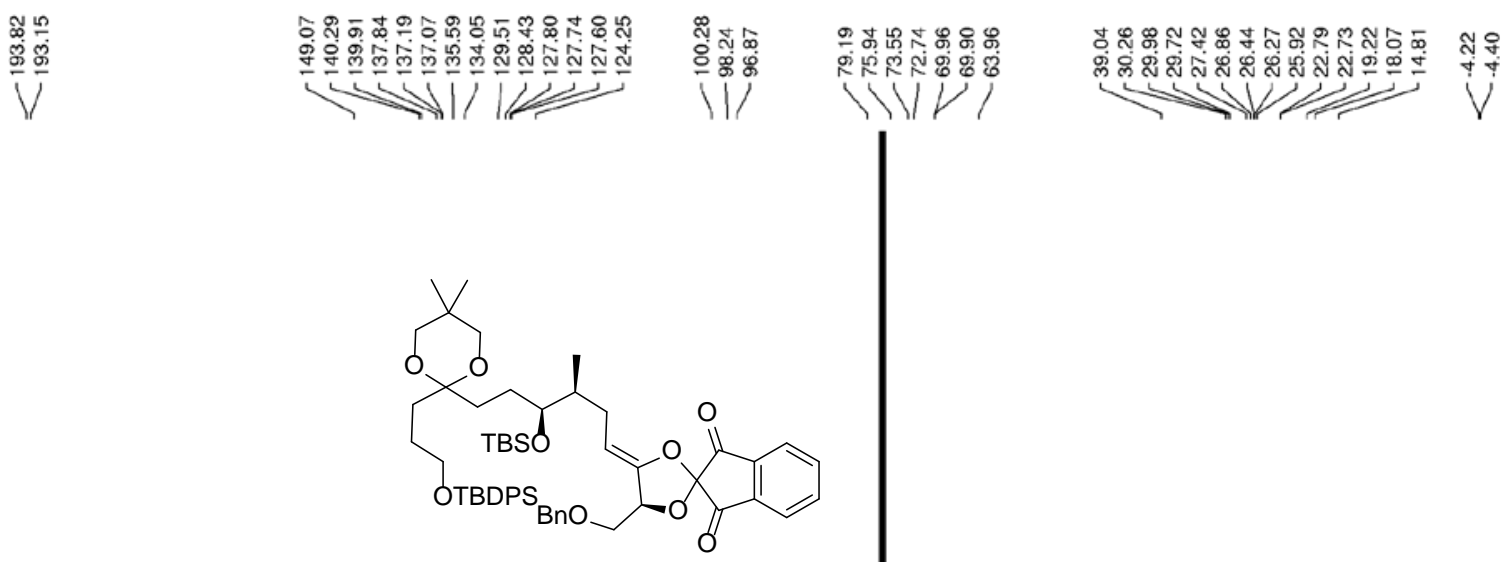

13

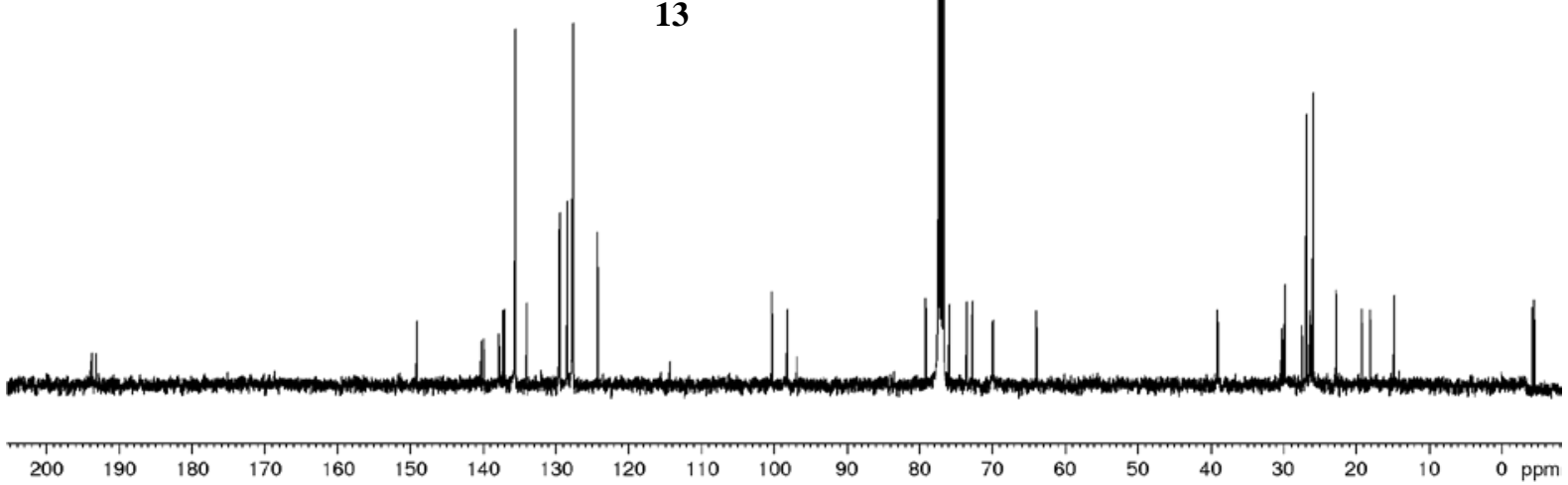



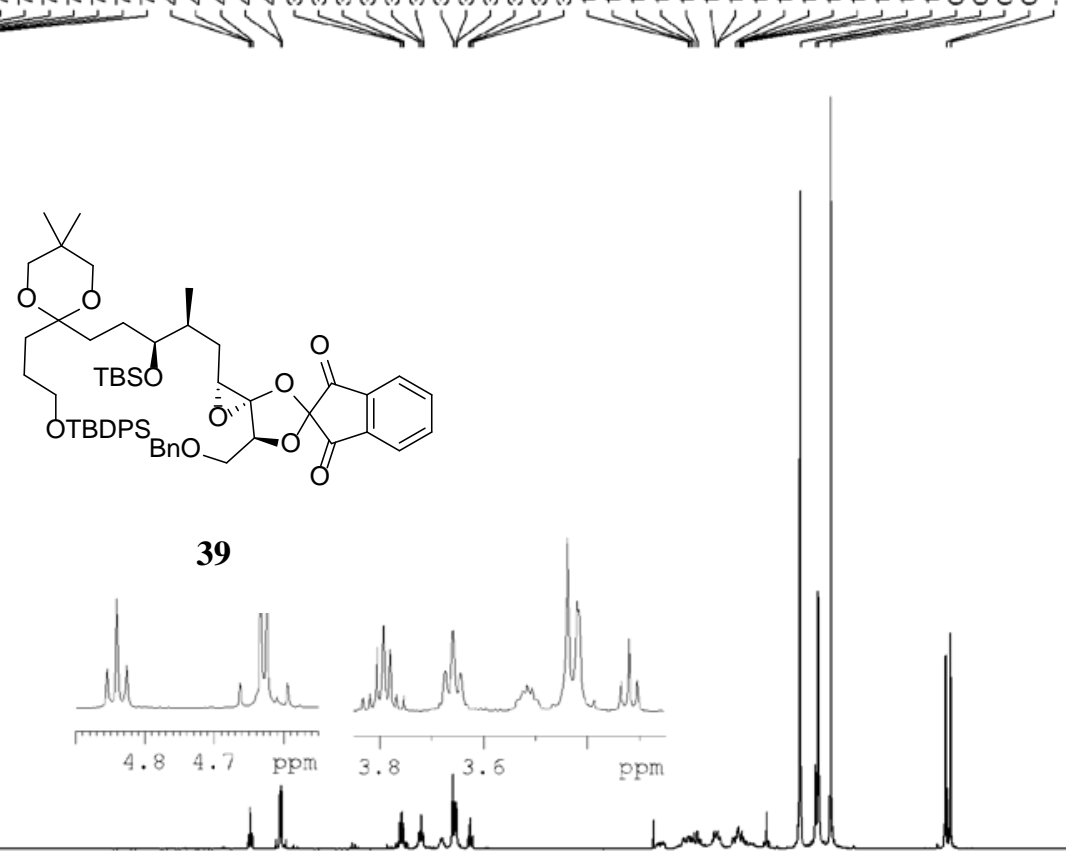

39

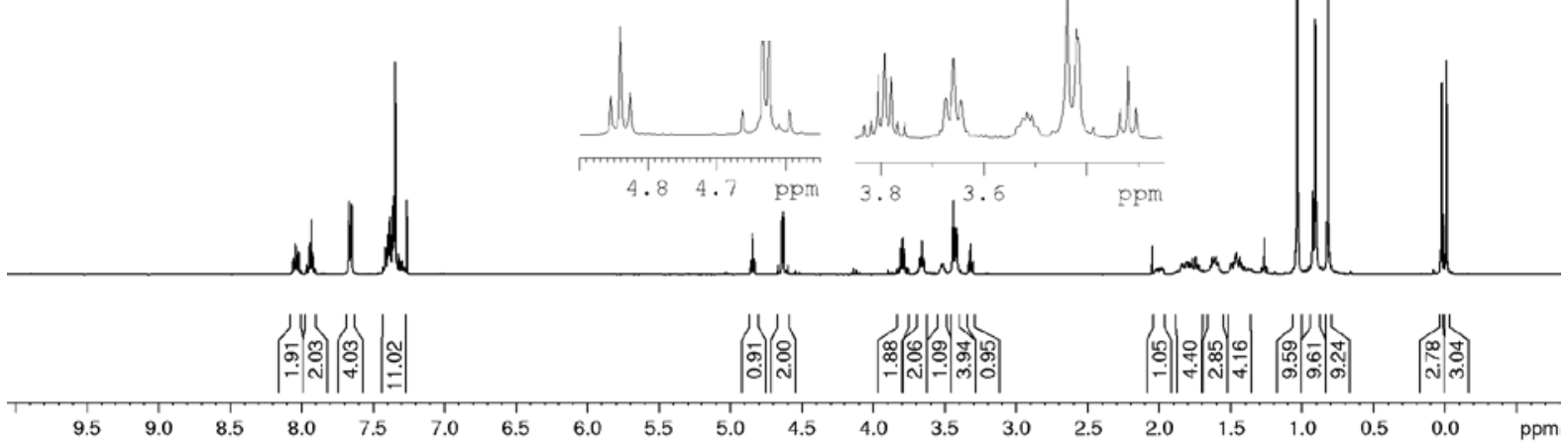

Compound 39, ${ }^{13} \mathrm{C}\left\{{ }^{1} \mathrm{H}\right\}\left(\mathrm{CDCl}_{3}, 100 \mathrm{MHz}\right)$
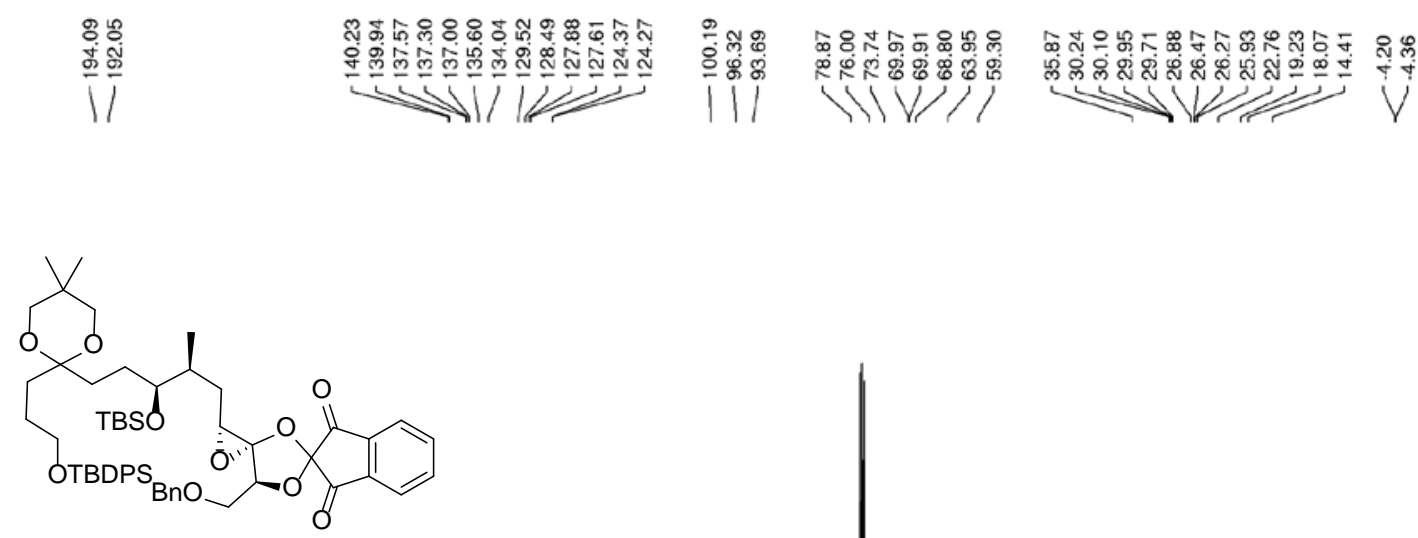

39

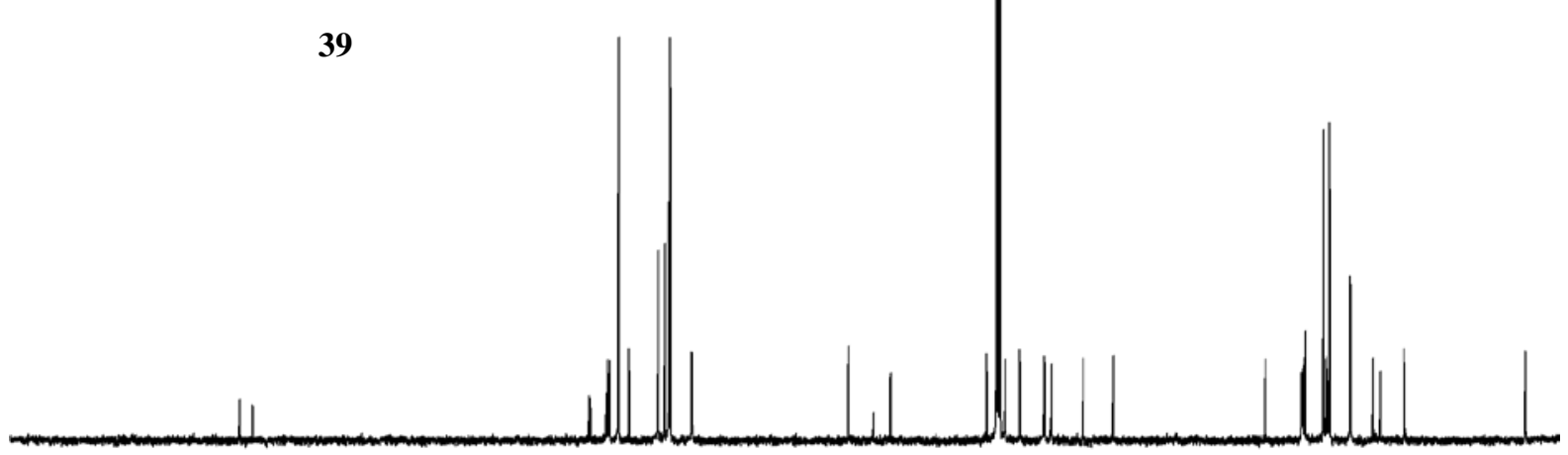

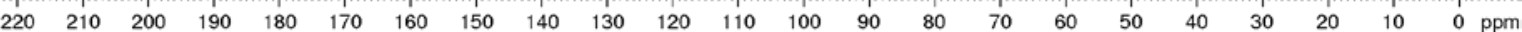




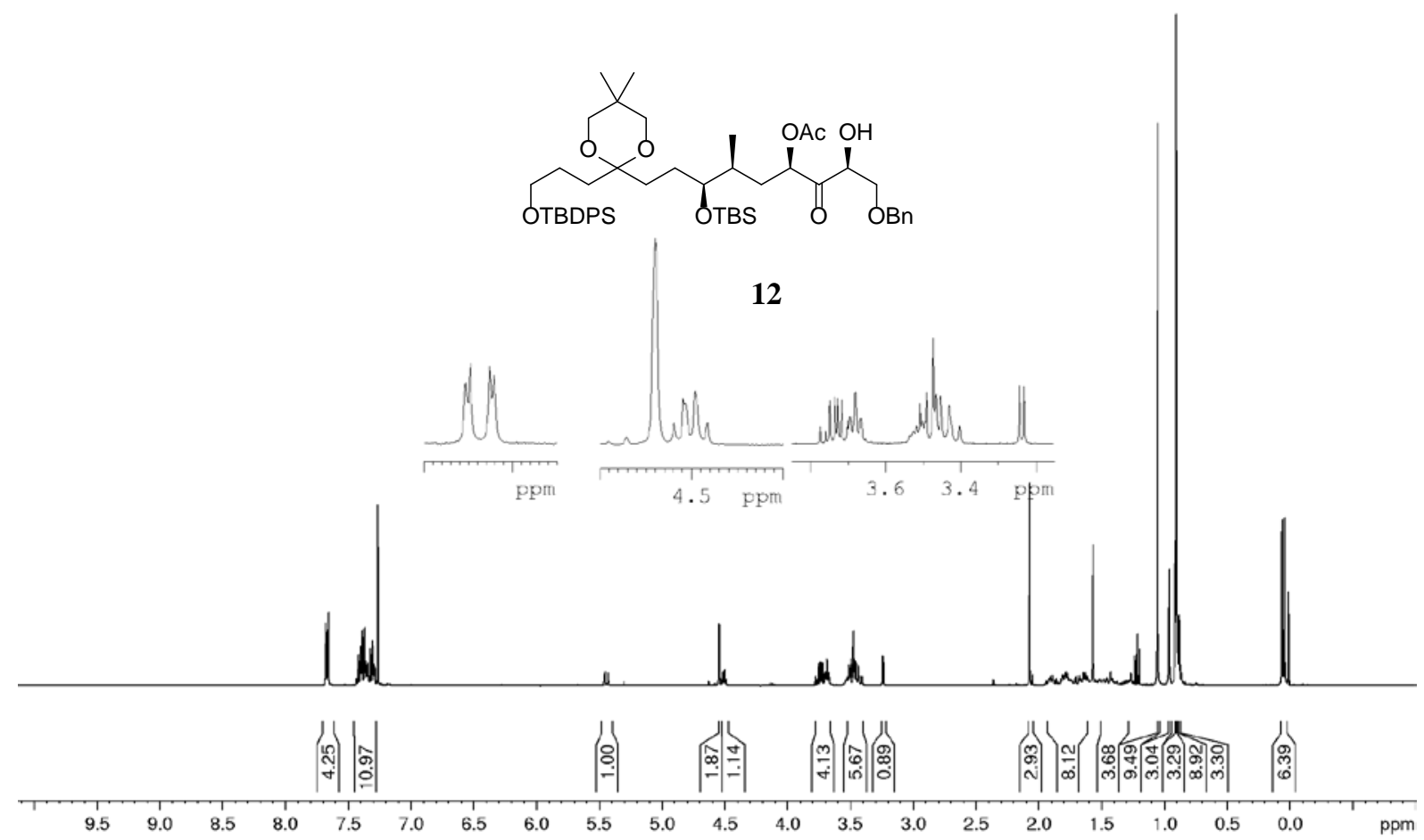

Compound 12, ${ }^{13} \mathrm{C}\left\{{ }^{1} \mathrm{H}\right\}\left(\mathrm{CDCl}_{3}, 100 \mathrm{MHz}\right)$
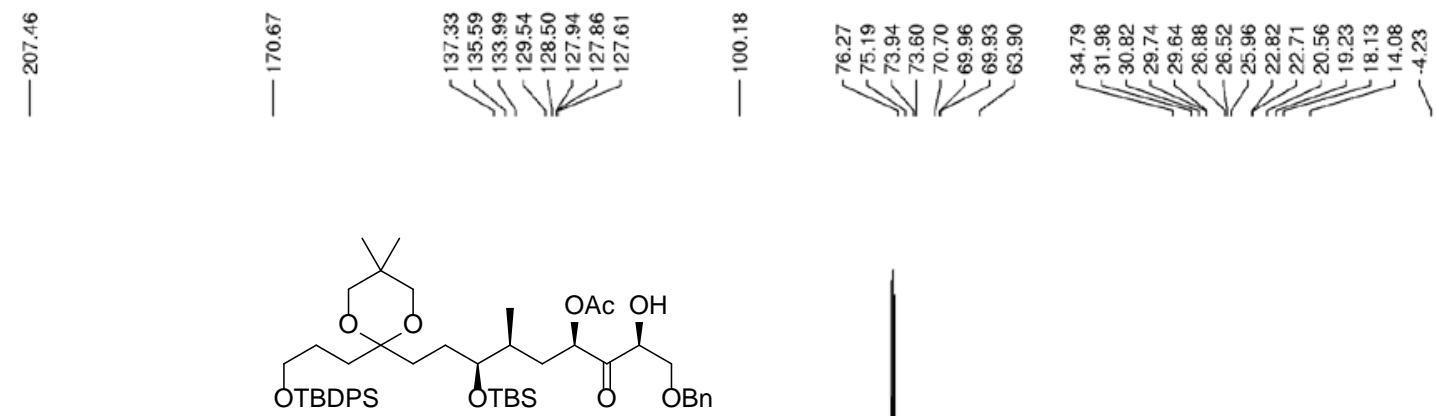

12

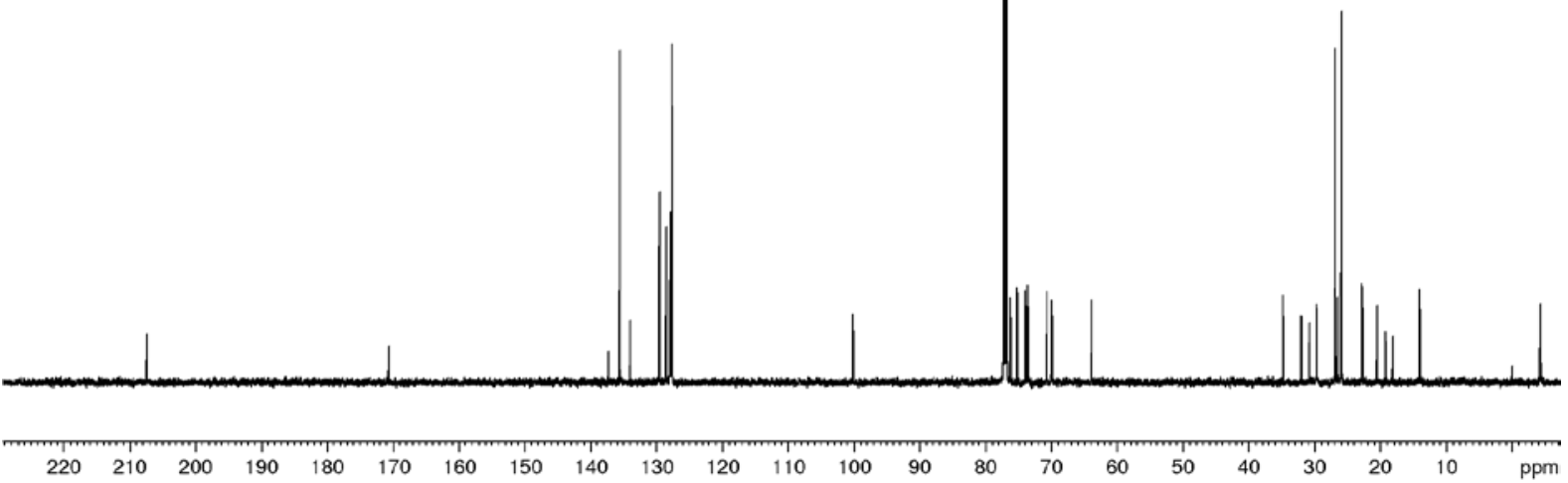


<smiles>CC(CC(O)C(C)C(=O)C(O)CCC(=O)CCCO)C(=O)O</smiles>

40
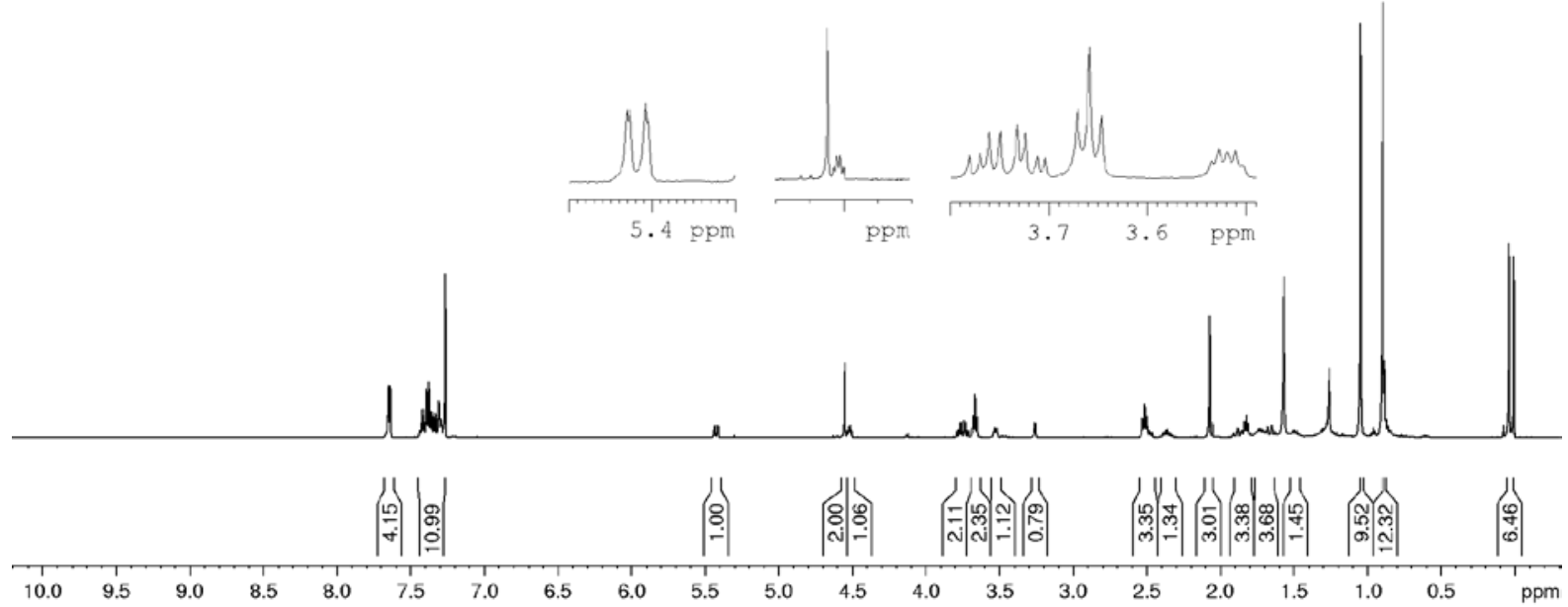

Compound 40, ${ }^{13} \mathrm{C}\left\{{ }^{1} \mathrm{H}\right\}\left(\mathrm{CDCl}_{3}, 100 \mathrm{MHz}\right)$
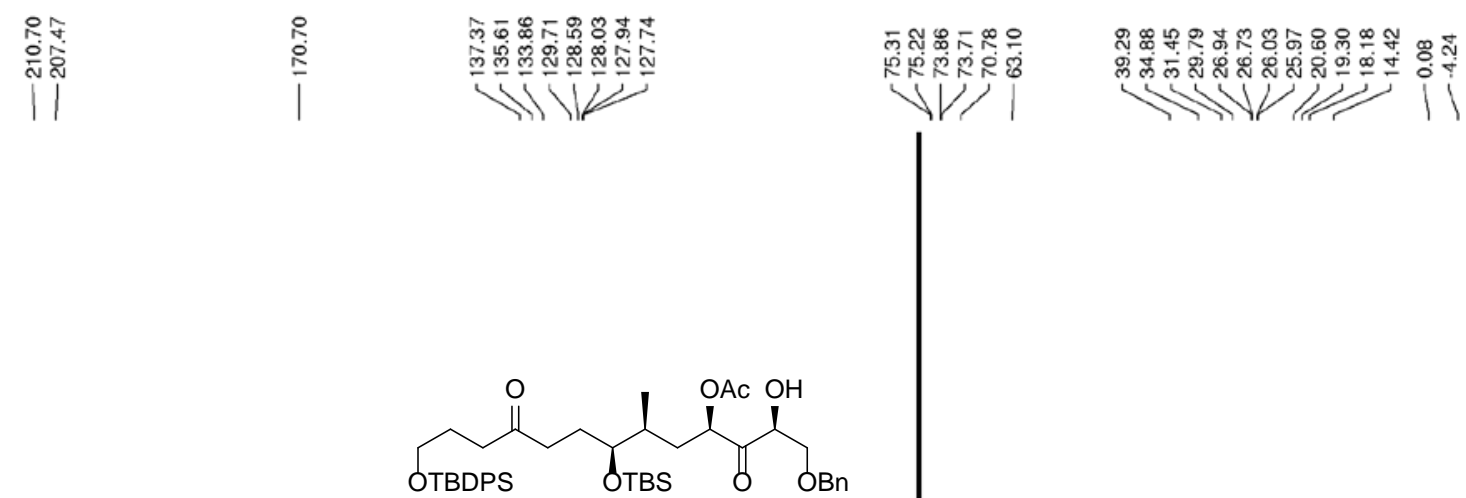

40

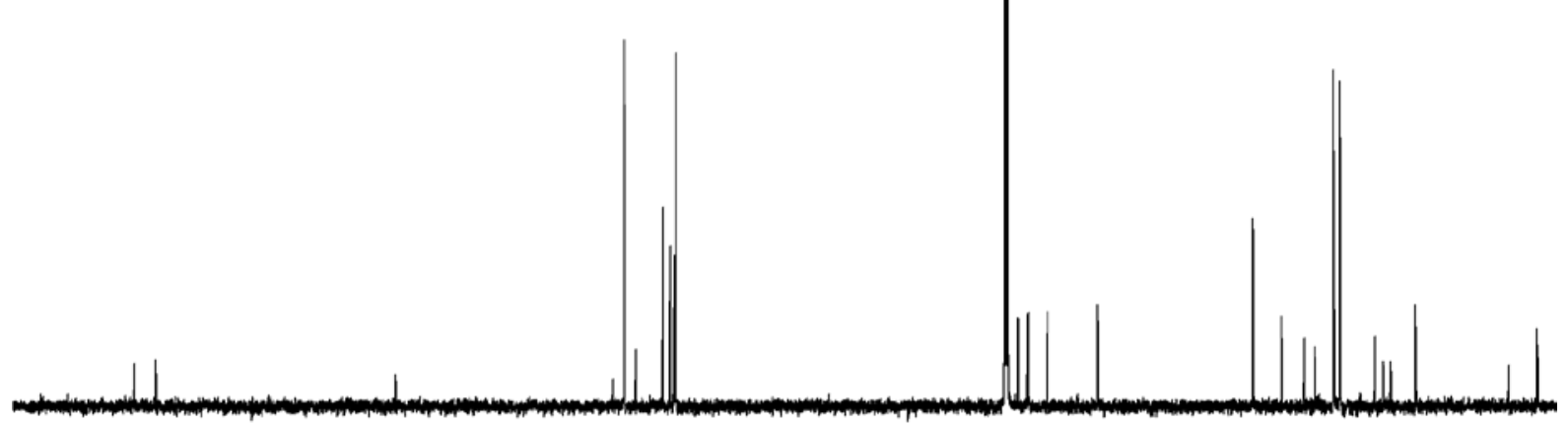

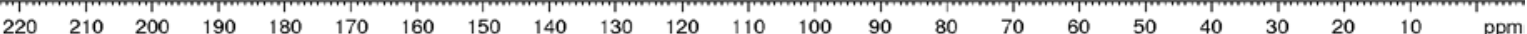




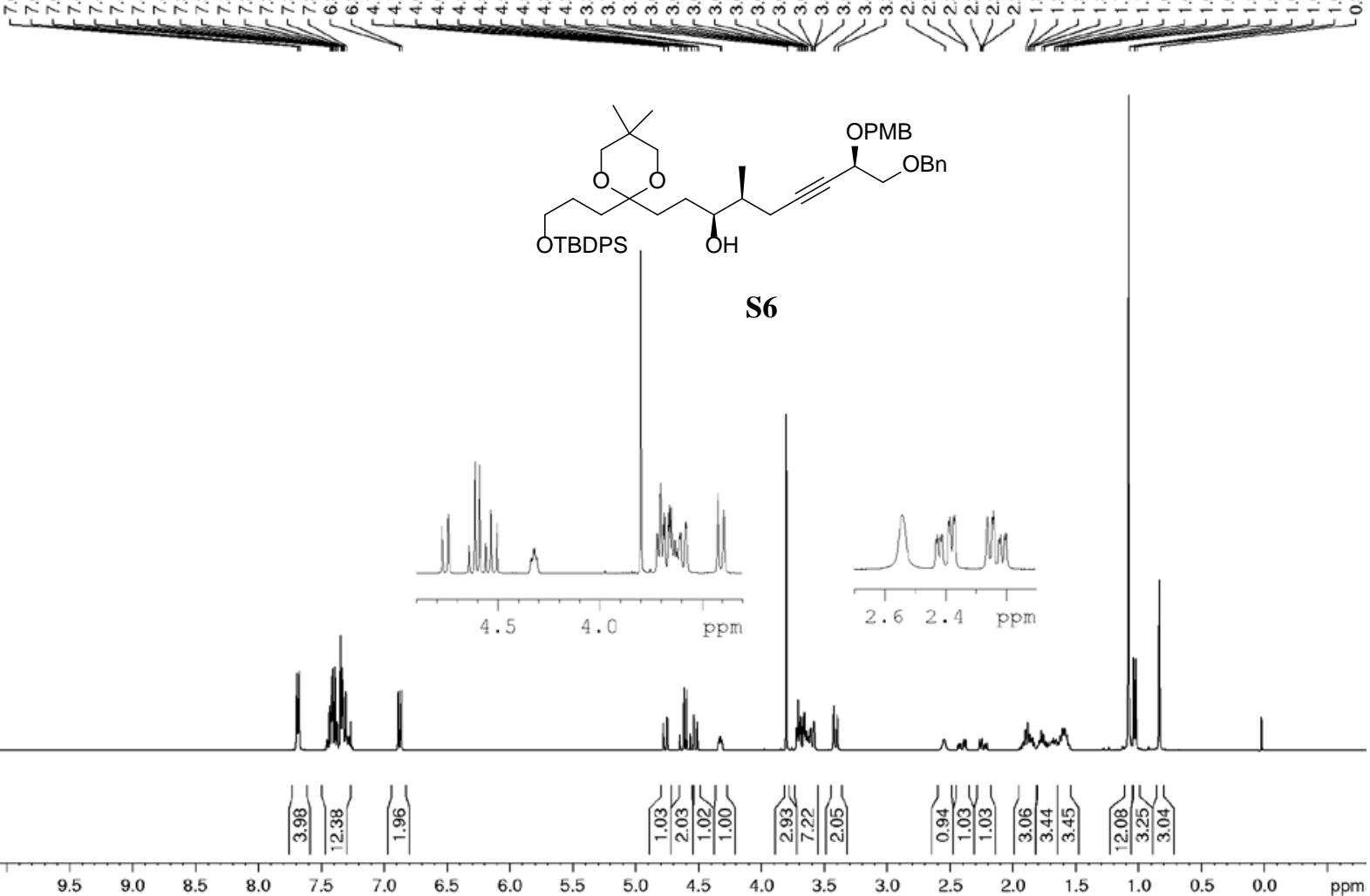

Compound S6, ${ }^{13} \mathrm{C}\left\{{ }^{1} \mathrm{H}\right\}\left(\mathrm{CDCl}_{3}, 100 \mathrm{MHz}\right)$

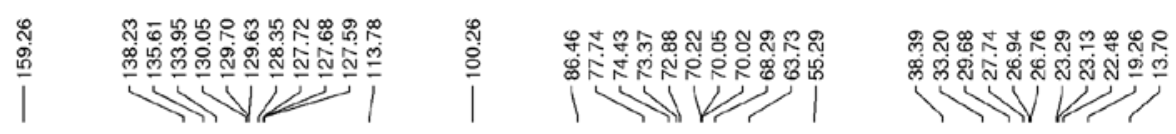<smiles>CC(CC#CC(CO[18OH])Cc1ccccc1)C(O)C(O)CCC1(CCC[Pb])OCC(C)(C)CO1</smiles>

S6

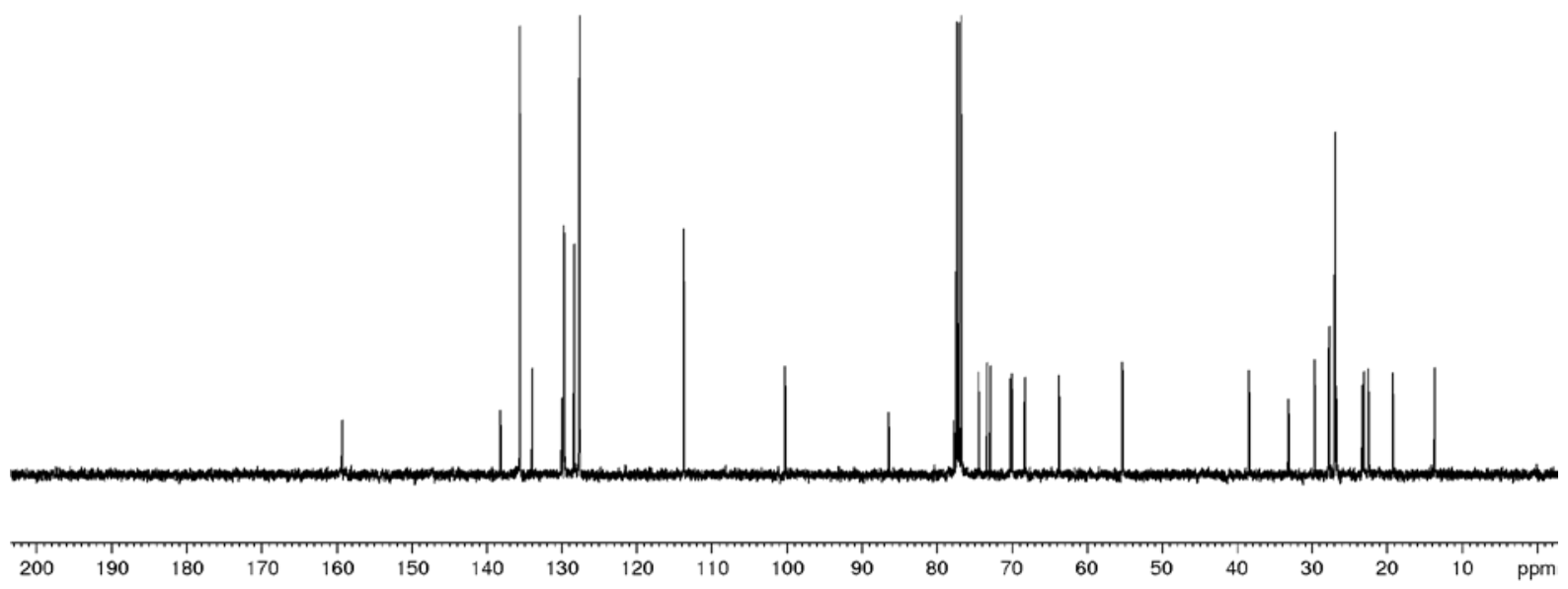




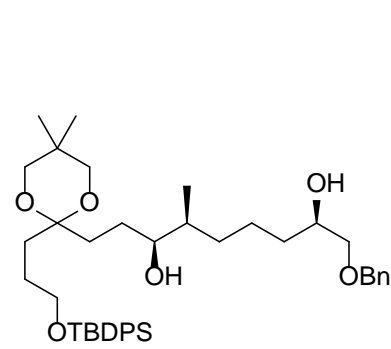

42

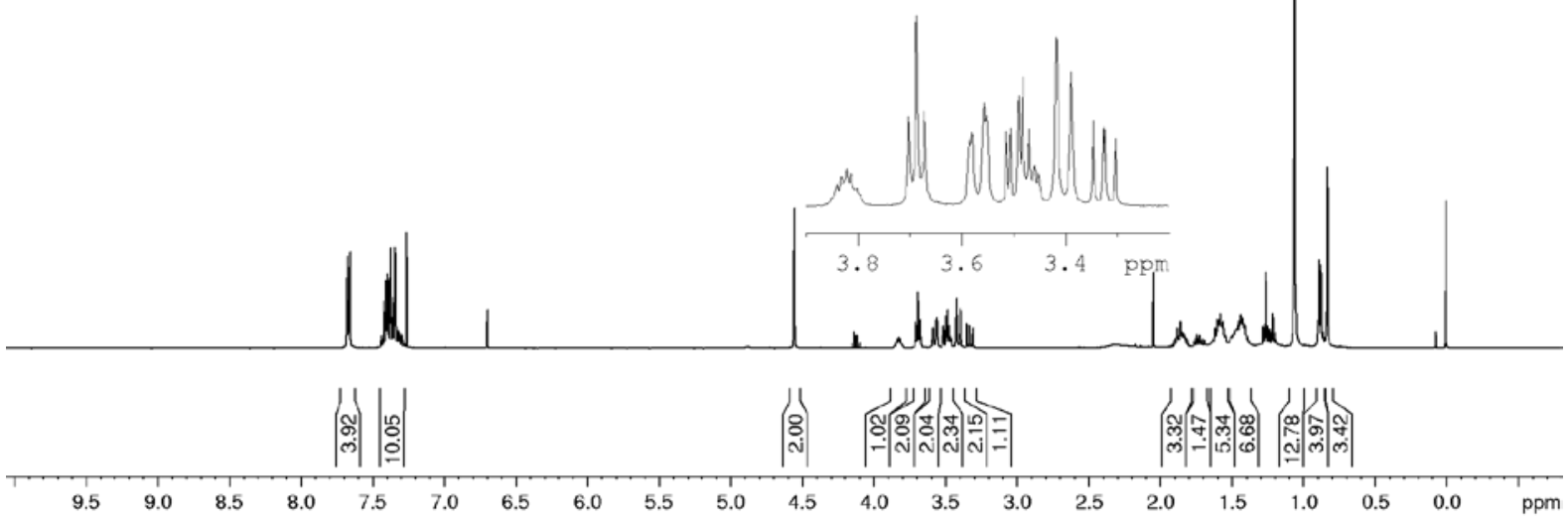

Compound $42,{ }^{13} \mathrm{C}\left\{{ }^{1} \mathrm{H}\right\}\left(\mathrm{CDCl}_{3}, 100 \mathrm{MHz}\right)$
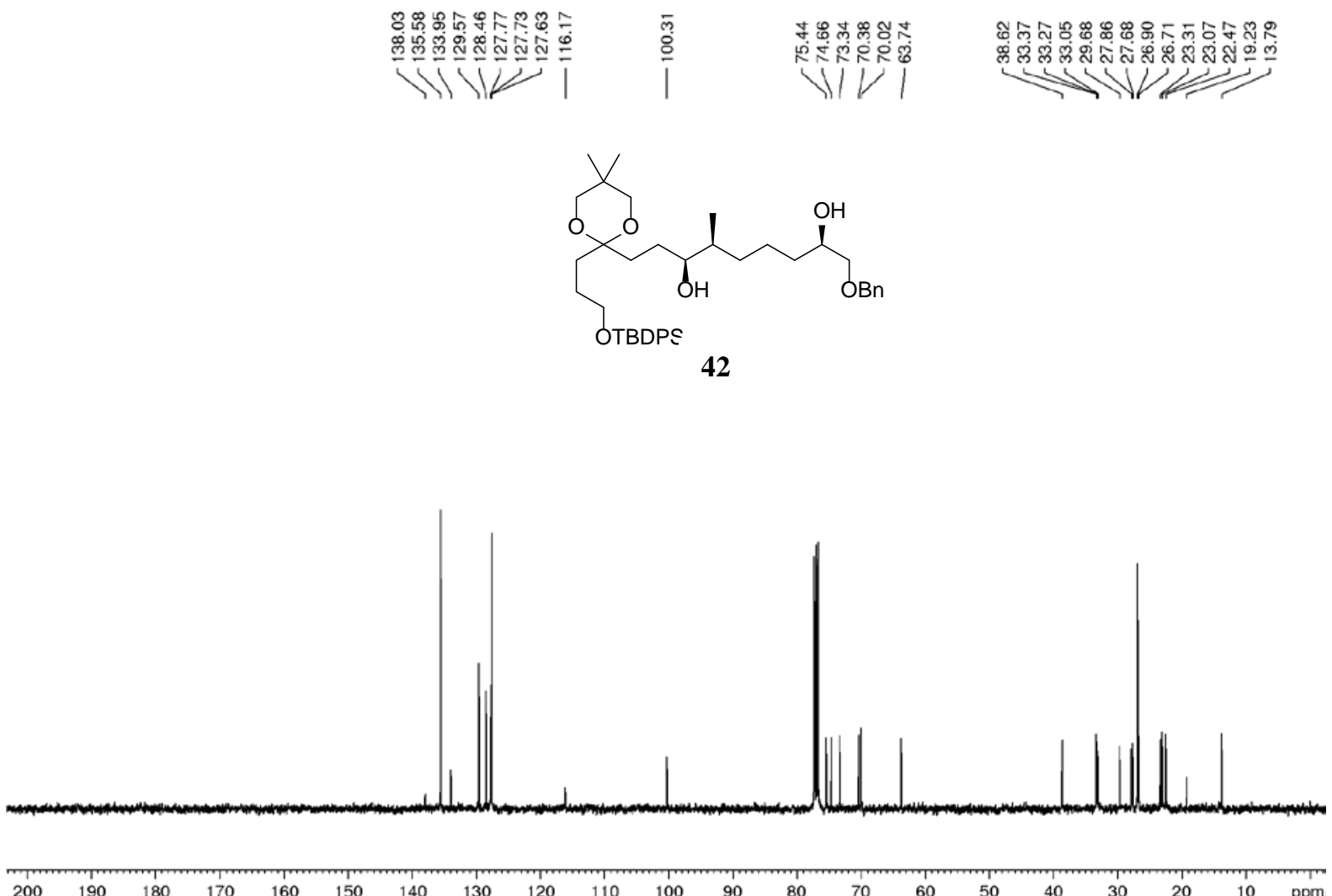
Determination of the enantiomer excess of crotylation product 24.<smiles>C=CC(C)C(CCCOC(C)(C)C)OC(=O)C(OC)(OC)c1ccccc1</smiles>

(3S,4S)-7-(4-Methoxybenzyloxy)-3-methylhept-1-en-4-yl

(R)-3,3,3-trifluoro-2-methoxy-2-phenylpropanoate 24a

To a solution of alcohol $24(10 \mathrm{mg}, 0.038 \mathrm{mmol})$ in dichloromethane $(0.5 \mathrm{~mL})$ was added a solution of $(R)-(+)-$ $\alpha$-methoxy- $\alpha$-trifluoromethylphenylacetic acid $(13 \mathrm{mg}, 0.058 \mathrm{mmol})$ in dichloromethane $(0.5 \mathrm{~mL})$, followed by $\mathrm{N}, \mathrm{N}$-dicyclohexylcarbodiimide (20 mg, $0.095 \mathrm{mmol}$ ) and 4-dimethylaminopyridine (0.92 mg, $0.0076 \mathrm{mmol})$. The reaction mixture was stirred for $16 \mathrm{~h}$ then filtered from a plug of Celite and concentrated in vacuo. Purification by flash chromatography using petroleum ether/ethyl acetate (3:1) as eluent afforded the title product 24a (18.4 mg, quant.) in $92 \%$ de as a colourless oil. IR $v_{\max }$ (neat) $/ \mathrm{cm}^{-1}: 2952,2853,1742,1513,1246,1167,1017,718 ;{ }^{1} \mathrm{H}$ NMR (400 MHz, $\left.\mathrm{CDCl}_{3}\right): \delta 7.57-7.53(\mathrm{~m}, 2 \mathrm{H}), 7.41-7.34(\mathrm{~m}, 3 \mathrm{H}), 7.27-7.21(\mathrm{~m}, 2 \mathrm{H}), 6.90-6.85(\mathrm{~m}, 2 \mathrm{H}), 5.64$ (ddd, $J=17.4,10.5,7.2 \mathrm{~Hz}, 1 \mathrm{H}), 5.10-5.04$ (m, 1H), 5.01-4.93 (m, 2H), 4.41 (s, 2H), 3.81 (s, 3H), 3.55-3.52 (m, 3H), 3.47-3.36 (m, 2H), 2.53-2.42 (m, 1H), 1.81-1.52 (m, 4H), 0.95 (d, $J=6.9 \mathrm{~Hz}, 3 \mathrm{H})$; ${ }^{13} \mathrm{C}$ NMR $(100 \mathrm{MHz}$, $\left.\mathrm{CDCl}_{3}\right): \delta 166.4,159.3,139.1,132.4,130.7,129.7,129.4(2 \times \mathrm{C}), 128.5(2 \times \mathrm{C}), 127.6(2 \times \mathrm{C}), 125.0,122.1,115.8$, $113.9(2 \times \mathrm{C}), 80.1,72.6,69.4,55.6,55.4,40.7,28.0,25.7,14.8 ; \mathrm{HRMS}$ (ESI/Q-TOF) m/z: [M+Na]+ Calcd for C26H31F3NaO5 503.2016; Found: 503.2013.

Compound 24a, ${ }^{1} \mathrm{H}$ NMR ( $\left.\mathrm{CDCl}_{3}, 400 \mathrm{MHz}\right)$

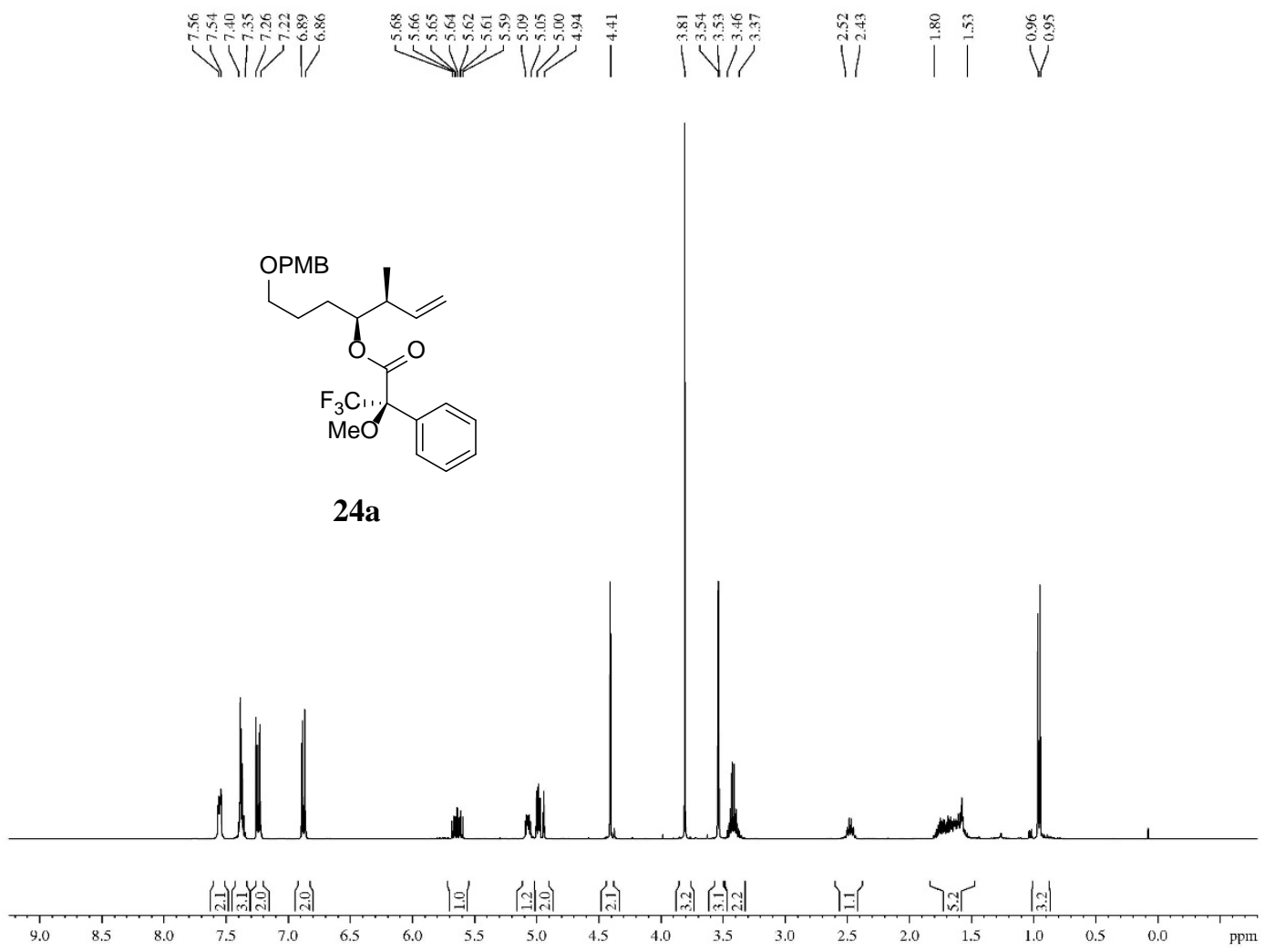


Compound 24a, ${ }^{13} \mathrm{C}\left\{{ }^{1} \mathrm{H}\right\}\left(\mathrm{CDCl}_{3}, 100 \mathrm{MHz}\right)$
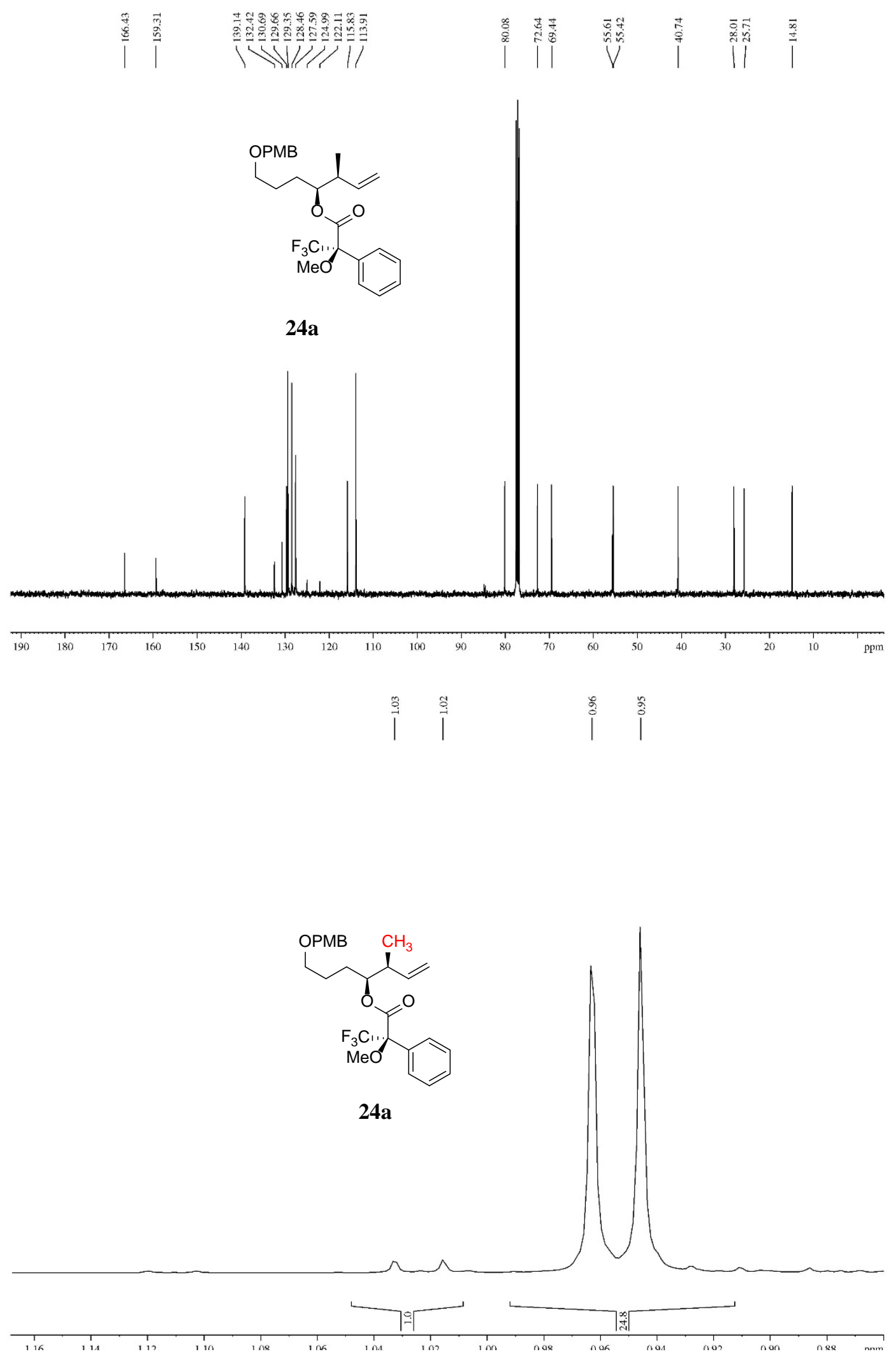
<smiles>C=CC(C)C(CCCOC(C)(C)C)OC(=O)C(C)(C)c1ccccc1</smiles>

To a solution of alcohol $24(10 \mathrm{mg}, 0.038 \mathrm{mmol})$ in dichloromethane $(0.5 \mathrm{~mL})$ was added a solution of $(S)-(-)-\alpha-$ methoxy- $\alpha$-trifluoromethylphenylacetic acid $(13 \mathrm{mg}, 0.058 \mathrm{mmol})$ in dichloromethane $(0.5 \mathrm{~mL})$, followed by N,N'-dicyclohexylcarbodiimide (20 mg, $0.095 \mathrm{mmol}$ ) and 4-dimethylaminopyridine (0.92 mg, $0.0076 \mathrm{mmol}$ ). The reaction mixture was stirred for $16 \mathrm{~h}$ then filtered through a plug of Celite and concentrated in vacuo. Purification by flash chromatography using petroleum ether/ethyl acetate (3:1) as eluent afforded the title product $24 \mathbf{b}$ (17.8 $\mathrm{mg}, 98 \%$ ) in $92 \%$ de as a colourless oil. IR $v_{\max }$ (neat) $/ \mathrm{cm}^{-1}: 2952,2853,1742,1513,1247,1168,1018,718 ;{ }^{1} \mathrm{H}$ NMR (400 MHz, $\left.\mathrm{CDCl}_{3}\right): \delta$ 7.57-7.53 (m, 2H), 7.44-7.34 (m, 3H), 7.25-7.20 (m, 2H), 6.90-6.84 (m, $\left.2 \mathrm{H}\right), 5.75$ (ddd, $J=17.1,10.5,6.8 \mathrm{~Hz}, 1 \mathrm{H}), 5.11-5.02$ (m, 3H), 4.37 (s, 2H), 3.81 (s, 3H), 3.54-3.52 (m, 3H), 3.42-3.30 (m, 2H), 2.58-2.48 (m, 1H), 1.76-1.40 (m, 4H), 1.02 (d, $J=7.0 \mathrm{~Hz}, 3 \mathrm{H}) ;{ }^{13} \mathrm{C}$ NMR (100 MHz, $\left.\mathrm{CDCl}_{3}\right): \delta 166.5$, 159.3, 139.3, 132.4, 130.7, 129.7, 129.3 (2×C), 128.5 (2×C), 127.7 (2×C), 125.0, 122.1, 115.9, $113.9(2 \times \mathrm{C}), 80.2$, 72.6, 69.4, 55.6, 55.4, 40.7, 27.7, 25.4, 14.9; HRMS (ESI/Q-TOF) m/z: [M+Na]+ Calcd for C26H31F3NaO5 503.2016; Found: 503.2013.

Compound 24b, ${ }^{1} \mathrm{H}$ NMR $\left(\mathrm{CDCl}_{3}, 400 \mathrm{MHz}\right)$

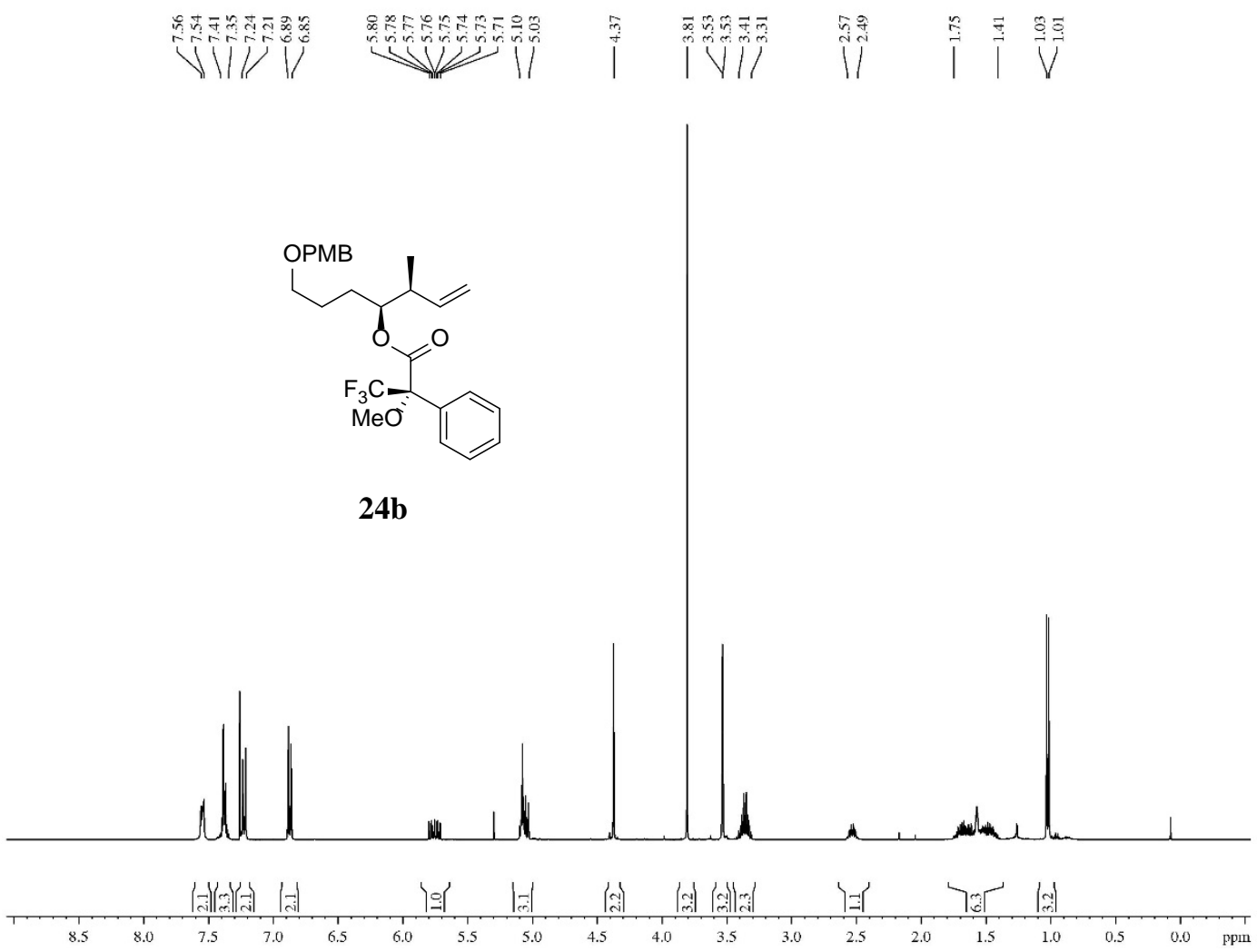


Compound 24b, ${ }^{13} \mathrm{C}\left\{{ }^{1} \mathrm{H}\right\}\left(\mathrm{CDCl}_{3}, 100 \mathrm{MHz}\right)$

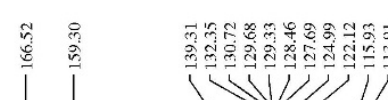

$1+1$

MII/1)

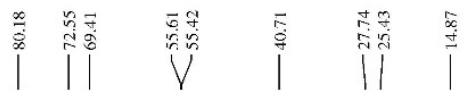

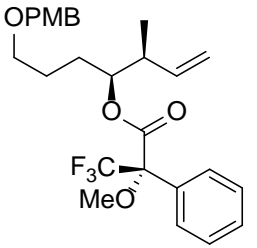

24b
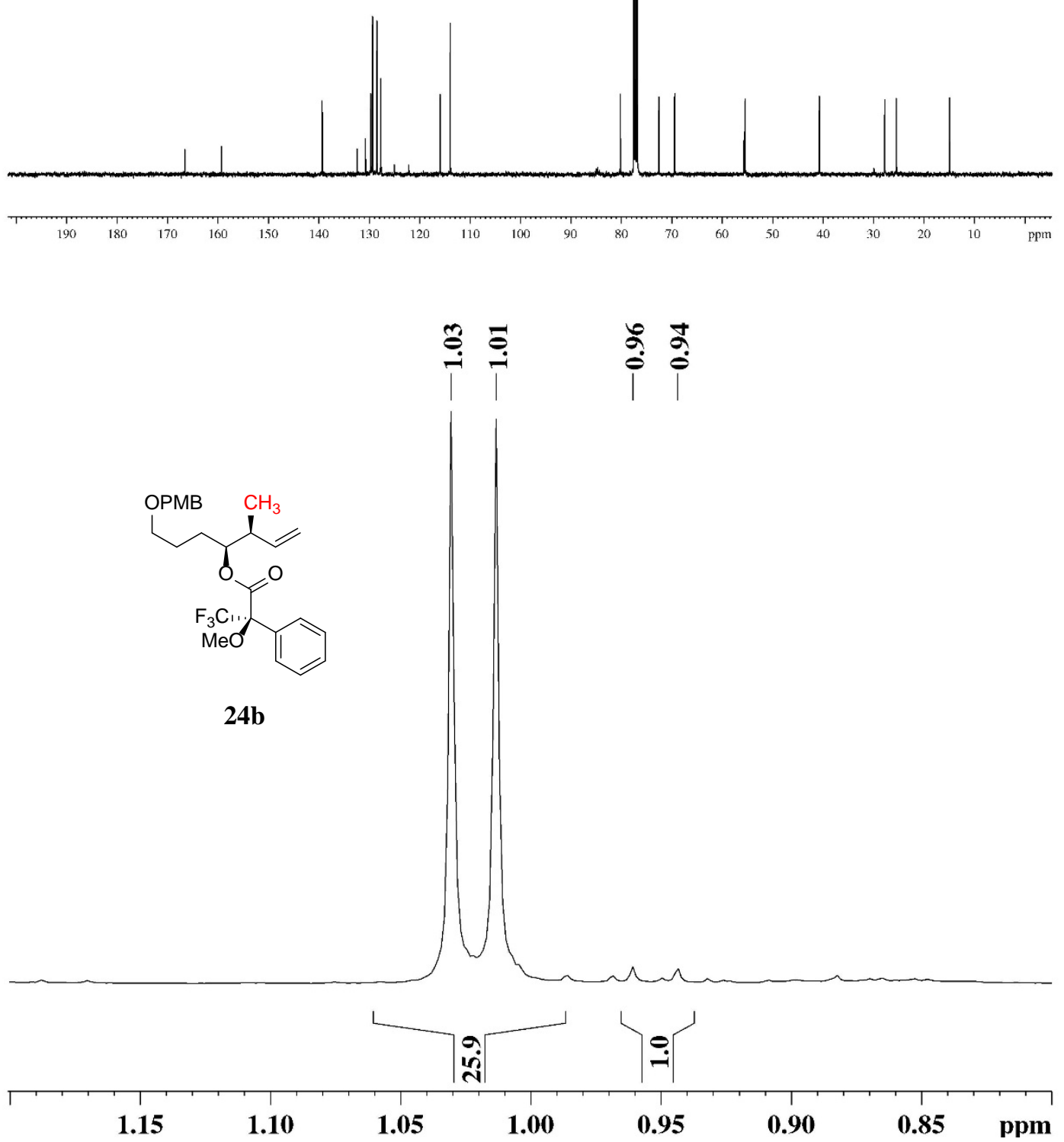
Conditions attempted for the cyclisation of compound $\mathbf{4 0 .}$

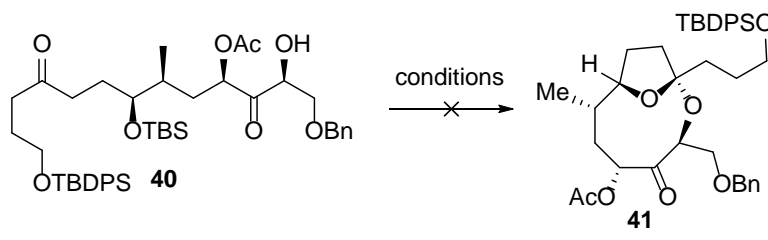

\begin{tabular}{|c|c|c|}
\hline Entry & Conditions & Results \\
\hline 1 & $\mathrm{CSA}$ (0.1 eq.), $\mathrm{MeCN}, 50^{\circ} \mathrm{C}$ & Decomposition \\
\hline 2 & PPTSA (0.2 eq.), MeCN, rt & Starting material \\
\hline 3 & PPTSA (0.2 eq.), $\mathrm{MeCN}, 50^{\circ} \mathrm{C}$ & Slow decomposition \\
\hline 4 & $\mathrm{CSA}$ (0.5 eq.), $\mathrm{MeCN}, 0^{\circ} \mathrm{C}$ to rt & Slow decomposition \\
\hline 5 & $\mathrm{CSA}$ (0.1 eq.), $\mathrm{CH}_{2} \mathrm{Cl}_{2}, \mathrm{rt}$ & Starting material \\
\hline 6 & $\mathrm{HCl}(1 \mathrm{M})$ (0.1 eq.), $\mathrm{MeCN}, \mathrm{rt}$ & Decomposition \\
\hline 7 & CSA (0.2 eq.), $\mathrm{MeOH}, \mathrm{rt}$ & Decomposition \\
\hline 8 & HF-py (0.5 eq.), MeCN, rt & Slow decomposition \\
\hline 9 & TBAF/AcOH (1:1, 1 eq.), THF, rt & $\begin{array}{c}\text { Deprotection of } \\
\text { TBDPS was observed }\end{array}$ \\
\hline 10 & $\mathrm{TBAF} / \mathrm{AcOH}$ (1:1, 1 eq.), THF, $50^{\circ} \mathrm{C}$ & Decomposition \\
\hline
\end{tabular}

